\title{
ABJM quantum spectral curve and Mellin transform
}

\author{
R.N. Lee ${ }^{a}$ and A.I. Onishchenko ${ }^{b, c, d}$ \\ ${ }^{a}$ Budker Institute of Nuclear Physics, \\ Novosibirsk, Russia \\ ${ }^{b}$ Bogoliubov Laboratory of Theoretical Physics, Joint Institute for Nuclear Research, \\ Dubna, Russia \\ ${ }^{c}$ Moscow Institute of Physics and Technology State University, \\ Dolgoprudny, Russia \\ ${ }^{d}$ Skobeltsyn Institute of Nuclear Physics, Moscow State University, \\ Moscow, Russia \\ E-mail: r.n.lee@inp.nsk.su, onish@theor.jinr.ru
}

ABSTRACT: The present techniques for the perturbative solution of quantum spectral curve problems in $\mathcal{N}=4 \mathrm{SYM}$ and ABJM models are limited to the situation when the states quantum numbers are given explicitly as some integer numbers. These techniques are sufficient to recover full analytical structure of the conserved charges provided that we know a finite basis of functions in terms of which they could be written explicitly. It is known that in the case of $\mathcal{N}=4 \mathrm{SYM}$ both the contributions of asymptotic Bethe ansatz and wrapping or finite size corrections are expressed in terms of the harmonic sums. However, in the case of ABJM model only the asymptotic contribution can still be written in the harmonic sums basis, while the wrapping corrections part can not. Moreover, the generalization of harmonic sums basis for this problem is not known. In this paper we present a Mellin space technique for the solution of multiloop Baxter equations, which is the main ingredient for the solution of corresponding quantum spectral problems, and provide explicit results for the solution of ABJM quantum spectral curve in the case of twist 1 operators in $s l(2)$ sector for arbitrary spin values up to four loop order with explicit account for wrapping corrections. It is shown that the result for anomalous dimensions could be expressed in terms of harmonic sums decorated by the fourth root of unity factors, so that maximum transcendentality principle holds.

Keywords: AdS-CFT Correspondence, Chern-Simons Theories, Integrable Field Theories, Supersymmetric Gauge Theory

ARXIV EPRINT: 1712.00412 


\section{Contents}

1 Introduction 1

2 ABJM quantum spectral curve 2

3 Perturbative solution of ABJM QSC 5

3.1 Leading order 6

$\begin{array}{lll}3.2 & \text { Next-to-leading order } & 7\end{array}$

4 Solution of Baxter equations with Mellin transform 9

$\begin{array}{ll}4.1 \text { Homogeneous solution } & 10\end{array}$

$\begin{array}{lll}4.2 & \text { Inhomogeneous solution } & 12\end{array}$

$\begin{array}{lll}4.2 .1 & \text { LO } & 12\end{array}$

$\begin{array}{lll}4.2 .2 & \text { NLO } & 13\end{array}$

5 Anomalous dimensions $\quad 16$

$\begin{array}{llr}6 & \text { Conclusion } & 18\end{array}$

$\begin{array}{lr}\text { A Mellin transformation } & 19\end{array}$

$\begin{array}{ll}\text { B Generating function and properties of Baxter polynomials } & 19\end{array}$

C Mellin transformations of arising functions $\quad 22$

\section{Introduction}

In the past time following the discovery of AdS/CFT duality [1-4] we faced a lot of progress in the study of integrable structures behind the quantum field theories with extended supersymmetry in dimensions greater then two, see for a review and introduction [5-10]. The most well understood theories are given by $\mathcal{N}=4 \mathrm{SYM}$ in four and $\mathcal{N}=6$ super Chern-Simons theory in three dimensions. The latter theory is more known as ABJM model [11]. In particular, different techniques from the world of integrable systems, such as worlsheet and spin-chain S-matrices [12-20], Asymptotic Bethe Ansatz (ABA) [16, 21-26] and Thermodynamic Bethe Ansatz (TBA) [27-30] as well as $Y$ and $T$-systems [31-37] were shown to be very useful for the computation of conformal spectrum of these theories. The integrability based methods were also applied to the study of quark-antiquark potential [3841], expectation values of polygonal Wilson loops at strong coupling and beyond [42-47], eigenvalues of BFKL kernel [48-50], structure constants [51-54] and one-point functions of operators in the defect conformal field theory [55-57]. 
Recently, the detailed study of TBA equations for $\mathcal{N}=4$ SYM and ABJM models resulted in the discovery of very effective Quantum Spectral Curve (QSC) formulation for these models [58-63]. The latter gives an alternative reformulation of TBA equations as a nonlinear Riemann-Hilbert problem. The iterative procedure for perturbative solution of the mentioned Riemann-Hilbert problems for these theories at weak coupling was finally proposed in [64, 65]. However, the presented technique is limited to the situation when the states quantum numbers are given explicitly as some integer numbers. It is sufficient for the recovery of full analytical structure of the conserved charges in the respective spin chains provided that we know a finite basis of functions in terms of which they could be written explicitly. It is known that in the case of $\mathcal{N}=4 \mathrm{SYM}$ both the contributions of asymptotic Bethe ansatz and wrapping or finite size corrections for twist-2 and twist-3 operators are expressed in terms of the harmonic sums [66-76]. However, in the case of ABJM model it was possible to express in the harmonic sums basis only the asymptotic contribution, while the wrapping corrections part misses such a representation. Moreover, the generalization of harmonic sums basis for this problem is not known at present [65, 7782]. In this paper we present a Mellin space technique for the solution of multiloop Baxter equations, which is the main ingredient for the solution of corresponding quantum spectral problems, and provide explicit results for the solution of ABJM quantum spectral curve in the case of twist 1 operators in $s l(2)$ sector for arbitrary spin values up to four loop order with explicit account for wrapping corrections. The results for anomalous dimensions we obtained could be further expressed in terms of harmonic sums decorated by the fourth root of unity factors.

The present paper is organized as follows. In the next section we remind the reader the formulation of ABJM quantum spectral curve. Section 3 contains the solution of ABJM QSC in the case of twist 1 operators in $\operatorname{sl}(2)$ sector up to four loop order. Next, in section 4 we present the details of solution of corresponding Baxter equations with Mellin space technique. Section 5 contains the discussion of our results for anomalous dimensions of twist 1 operators up to four loop order and for arbitrary values of spin variable. Finally, in section 6 we come with our conclusion. Appendices and Mathematica notebooks contain some details of our calculation.

\section{ABJM quantum spectral curve}

ABJM is a three-dimensional $\mathcal{N}=6$ Chern-Simons theory with product gauge group $\mathrm{U}(N) \times \hat{\mathrm{U}}(N)$ at levels $\pm k$. The field content of the theory is given by two gauge fields $A_{\mu}$ and $\hat{A}_{\mu}$, four complex scalars $Y^{A}$ and four Weyl spinors $\psi_{A}$. The matter fields transform in the bi-fundamental representation of the gauge group. The global symmetry group of ABJM theory for Chern-Simons level $k>2$ is given by orthosymplectic supergroup $\operatorname{OSp}(6 \mid 4)[11,83]$ and the "baryonic" $\mathrm{U}(1)_{b}[83]$. In the present paper we will be interested in anomalous dimensions of $s l(2)$-like states given by single-trace operators of the form [84]:

$$
\operatorname{tr}\left[D_{+}^{S}\left(Y^{1} Y_{4}^{\dagger}\right)^{L}\right] .
$$


In fact in what follows we will restrict ourselves with only twist $1(L=1)$ operators. It should be noted that these operators characterized by Dynkin labels $[L+S, S ; L, 0, L]$ do not form a closed subsector by themselves but belong to a wider $\operatorname{OSp}(2 \mid 2)$ subsector. At the moment the most advanced method to deal with spin chain spectral problems arising in the study if $\mathrm{AdS}_{d+1} / \mathrm{CFT}_{d}$ duality is offered by quantum spectral curve (QSC) method. For the case of ABJM theory QSC formulation was introduced in [62, 63], see also [65].

The QSC method is another reformulation of Thermodynamic Bethe Ansatz (TBA) as a set of functional equations, such as $Y$ or $T$-systems. The QSC method or $\mathbf{P} \mu$-system is special in a sense, that it involves only a finite number of objects satisfying a set of nonlinear matrix Riemann-Hilbert equations. The $\mathbf{P} \mu$-system for ABJM consists of six functions $\mathbf{P}_{a}, a=1, \ldots, 6$ and an antisymmetric $6 \times 6$ matrix $\mu_{a b}$. Both $\mathbf{P}_{a}$ and $\mu_{a b}$ are functions of spectral parameter $u$. The $\mathbf{P}_{a}$ functions are defined on a Riemann sheet with a single cut running from $-2 h$ to $+2 h$ ( $h$ is ABJM QSC coupling constant), while functions $\mu_{a b}$ have an infinity of branch cuts at intervals $(-2 h,+2 h)+i n, n \in \mathbb{Z}$ and satisfy a simple relation

$$
\tilde{\mu}_{a b}(u)=\mu_{a b}(u+i)
$$

where $\tilde{f}$ here and in the following will denote a function $f$ analytically continued around one of the branch points on the real axis. It is important to mention that, in contrast to $\mathcal{N}=4$ SYM, ABJM QSC coupling constant $h$ is a nontrivial function of ABJM t'Hooft coupling constant $\lambda[25,85]$, which scales as $h \sim \lambda$ at small and as $h \sim \sqrt{\lambda / 2}$ at strong coupling constant. An important conjecture for the exact form of $h(\lambda)$ was made in $[86,87]$ by a comparison with the structure of localization results. The functions $\mathbf{P}_{a}$ and $\mu_{a b}$ satisfy the set of nonlinear constraints

$$
\begin{aligned}
& \mathbf{P}_{5} \mathbf{P}_{6}=1+\mathbf{P}_{2} \mathbf{P}_{3}-\mathbf{P}_{1} \mathbf{P}_{4}, \\
& \mu \chi \mu \chi=0,
\end{aligned}
$$

where nonzero entries of $6 \times 6$ symmetric matrix $\chi$ are given by

$$
\chi^{14}=\chi^{41}=-1, \quad \chi^{23}=\chi^{32}=1, \quad \chi^{56}=\chi^{65}=-1 .
$$

The fundamental Riemann-Hilbert relations for $\mathbf{P}_{a}$ and $\mu_{a b}$ functions are written as

$$
\begin{aligned}
\tilde{\mathbf{P}}_{a} & =\mathbf{P}_{a}-\mu_{a b} \chi^{b c} \mathbf{P}_{c} \\
\mu_{a b}-\tilde{\mu}_{a b} & =-\mathbf{P}_{a} \tilde{\mathbf{P}}_{b}+\mathbf{P}_{b} \tilde{\mathbf{P}}_{a} .
\end{aligned}
$$

It should be noted that, similar to the $\mathcal{N}=4$ SYM there is a complementary set of functions satisfying their Riemann-Hilbert relations, so called $\mathbf{Q} \omega$-system [62]. The $\mathbf{Q} \omega$-system is similar to $\mathbf{P} \mu$-system (2.3)-(2.7) with replacements

$$
\mathbf{P}_{a} \rightarrow \mathbf{Q}_{a}, \quad \mu_{a b} \rightarrow \omega_{a b} .
$$

The $\mathbf{Q}$ and $\omega$ functions have different cut structure however, see for details $[62,63]$ and $[58$, 59]. In the case of ABJM model it is convenient to parametrize $\mu_{a b}$ matrix in terms of 8 
functions $\nu_{i}, \bar{\nu}_{i}, i=1, \ldots, 4$ as $[62,63]$ :

$$
\mu_{a b}=\left(\begin{array}{cccccc}
0 & \nu_{1} \bar{\nu}_{1} & \nu_{2} \bar{\nu}_{2} & \bar{\nu}_{2} \nu_{3}-\bar{\nu}_{1} \nu_{4} & \nu_{1} \bar{\nu}_{2} & \bar{\nu}_{1} \nu_{2} \\
-\nu_{1} \bar{\nu}_{1} & 0 & \bar{\nu}_{2} \nu_{3}+\nu_{1} \bar{\nu}_{4} & \nu_{3} \bar{\nu}_{3} & \nu_{1} \bar{\nu}_{3} & \bar{\nu}_{1} \nu_{3} \\
-\nu_{2} \bar{\nu}_{2} & -\bar{\nu}_{2} \nu_{3}-\nu_{1} \bar{\nu}_{4} & 0 & \nu_{4} \bar{\nu}_{4} & -\bar{\nu}_{2} \nu_{4} & -\nu_{2} \bar{\nu}_{4} \\
\bar{\nu}_{1} \nu_{4}-\bar{\nu}_{2} \nu_{3} & -\nu_{3} \bar{\nu}_{3} & -\nu_{4} \bar{\nu}_{4} & 0 & -\bar{\nu}_{3} \nu_{4} & -\nu_{3} \bar{\nu}_{4} \\
-\nu_{1} \bar{\nu}_{2} & -\nu_{1} \bar{\nu}_{3} & \bar{\nu}_{2} \nu_{4} & \bar{\nu}_{3} \nu_{4} & 0 & \bar{\nu}_{2} \nu_{3}-\nu_{2} \bar{\nu}_{3} \\
-\bar{\nu}_{1} \nu_{2} & -\bar{\nu}_{1} \nu_{3} & \nu_{2} \bar{\nu}_{4} & \nu_{3} \bar{\nu}_{4} & \nu_{2} \bar{\nu}_{3}-\bar{\nu}_{2} \nu_{3} & 0
\end{array}\right)
$$

with an additional constraint: $\nu_{1} \bar{\nu}_{4}-\bar{\nu}_{1} \nu_{4}=\nu_{2} \bar{\nu}_{3}-\bar{\nu}_{2} \nu_{3}$. Here $\nu_{i}$ and $\bar{\nu}_{i}$ satisfy already periodic/anti-periodic constraints $(\sigma= \pm 1)$ :

$$
\tilde{\nu}_{i}=\sigma_{i} \nu_{i}^{[2]}=\sigma \nu_{i}^{[2]}
$$

where here and in what follows $f^{[n]}(u)=f(u+i n / 2)$. To describe anomalous dimensions of $s l(2)$-like states (2.1) it is enough to consider $\mathbf{P} \mu$-system reduced to symmetric, parity invariant states. The reduced $\mathbf{P} \mu$-system is identified by constraints $\mathbf{P}_{5}=\mathbf{P}_{6}=\mathbf{P}_{0}, \nu_{i}=\bar{\nu}_{i}$ and is written as $[62,63,65]$ :

$$
\begin{aligned}
\tilde{\nu}_{i} & =-\mathbf{P}_{i j} \chi^{j k} \nu_{k}, \\
\widetilde{\mathbf{P}}_{i j}-\mathbf{P}_{i j} & =\nu_{i} \tilde{\nu}_{j}-\nu_{j} \tilde{\nu}_{i},
\end{aligned}
$$

where

$$
\mathbf{P}_{i j}=\left(\begin{array}{cccc}
0 & -\mathbf{P}_{1} & -\mathbf{P}_{2} & -\mathbf{P}_{0} \\
\mathbf{P}_{1} & 0 & -\mathbf{P}_{0} & -\mathbf{P}_{3} \\
\mathbf{P}_{2} & \mathbf{P}_{0} & 0 & -\mathbf{P}_{4} \\
\mathbf{P}_{0} & \mathbf{P}_{3} & \mathbf{P}_{4} & 0
\end{array}\right), \quad \chi^{i j}=\left(\begin{array}{cccc}
0 & 0 & 0 & -1 \\
0 & 0 & 1 & 0 \\
0 & -1 & 0 & 0 \\
1 & 0 & 0 & 0
\end{array}\right)
$$

and

$$
\left(\mathbf{P}_{0}\right)^{2}=1-\mathbf{P}_{1} \mathbf{P}_{4}+\mathbf{P}_{2} \mathbf{P}_{3}
$$

In addition to the above constraints it is required [65], that $\mathbf{P}$ and $\nu$ functions have no poles and stay bounded at branch points. The quantum numbers of the states we are interested in, that is twist L, spin $\mathrm{S}$ and conformal dimension $\triangle$ are encoded in the behavior of $\mathbf{P}, \nu$ functions at large $u[62,63,65]$ :

$$
\begin{aligned}
& \mathbf{P}_{0-4} \simeq\left(A_{0} u^{0}, A_{1} u^{-L}, A_{2} u^{-L-1}, A_{3} u^{+L+1}, A_{4} u^{+L}\right) \\
& A_{1} A_{4}=-\frac{(\Delta-L+S)(\Delta-L-S+1)(\Delta+L-S+1)(\Delta+L+S)}{L^{2}(2 L+1)} \\
& A_{2} A_{3}=-\frac{(\Delta-L+S-1)(\Delta-L-S)(\Delta+L-S+2)(\Delta+L+S+1)}{(L+1)^{2}(2 L+1)}
\end{aligned}
$$

and

$$
\nu_{i} \sim\left(u^{\Delta-L}, u^{\Delta+1}, u^{\Delta}, u^{\Delta+L+1}\right) .
$$

The anomalous dimension $\gamma$ is given by $\gamma=\triangle-L-S$. 


\section{Perturbative solution of ABJM QSC}

For the perturbative solution of ABJM quantum spectral curve we use the same set of equations as in [65]. The latter easily follow ${ }^{1}$ from fundamental $\mathbf{P} \nu$-system (2.11)-(2.12) and are given by

$$
\begin{aligned}
& \frac{\nu_{1}^{[3]}}{\mathbf{P}_{1}^{[1]}}-\frac{\nu_{1}^{[-1]}}{\mathbf{P}_{1}^{[-1]}}-\sigma\left(\frac{\mathbf{P}_{0}^{[1]}}{\mathbf{P}_{1}^{[1]}}-\frac{\mathbf{P}_{0}^{[-1]}}{\mathbf{P}_{1}^{[-1]}}\right) \nu_{1}^{[1]}=-\sigma\left(\frac{\mathbf{P}_{2}^{[1]}}{\mathbf{P}_{1}^{[1]}}-\frac{\mathbf{P}_{2}^{[-1]}}{\mathbf{P}_{1}^{[-1]}}\right) \nu_{2}^{[1]}, \\
& \frac{\nu_{2}^{[3]}}{\mathbf{P}_{1}^{[1]}}-\frac{\nu_{2}^{[-1]}}{\mathbf{P}_{1}^{[-1]}}+\sigma\left(\frac{\mathbf{P}_{0}^{[1]}}{\mathbf{P}_{1}^{[1]}}-\frac{\mathbf{P}_{0}^{[-1]}}{\mathbf{P}_{1}^{[-1]}}\right) \nu_{2}^{[1]}=\sigma\left(\frac{\mathbf{P}_{3}^{[1]}}{\mathbf{P}_{1}^{[1]}}-\frac{\mathbf{P}_{3}^{[-1]}}{\mathbf{P}_{1}^{[-1]}}\right) \nu_{1}^{[1]},
\end{aligned}
$$

and

$$
\begin{aligned}
\sigma \nu_{1}^{[2]} & =\mathbf{P}_{0} \nu_{1}-\mathbf{P}_{2} \nu_{2}+\mathbf{P}_{1} \nu_{3}, \\
\sigma \nu_{2}^{[2]} & =-\mathbf{P}_{0} \nu_{2}+\mathbf{P}_{3} \nu_{1}+\mathbf{P}_{1} \nu_{4}, \\
\tilde{\mathbf{P}}_{2}-\mathbf{P}_{2} & =\sigma\left(\nu_{3} \nu_{1}^{[2]}-\nu_{1} \nu_{3}^{[2]}\right), \\
\tilde{\mathbf{P}}_{1}-\mathbf{P}_{1} & =\sigma\left(\nu_{2} \nu_{1}^{[2]}-\nu_{1} \nu_{2}^{[2]}\right), \\
\left(\nu_{1}+\sigma \nu_{1}^{[2]}\right)\left(\mathbf{p}_{0}-(h x)^{L}\right) & =\mathbf{p}_{2}\left(\nu_{2}+\sigma \nu_{2}^{[2]}\right)-\mathbf{p}_{1}\left(\nu_{3}+\sigma \nu_{3}^{[2]}\right), \\
\left(\nu_{2}+\sigma \nu_{2}^{[2]}\right)\left(\mathbf{p}_{0}+(h x)^{L}\right) & =\mathbf{p}_{3}\left(\nu_{1}+\sigma \nu_{1}^{[2]}\right)+\mathbf{p}_{1}\left(\nu_{4}+\sigma \nu_{4}^{[2]}\right),
\end{aligned}
$$

where

$$
x \equiv x(u)=\frac{u+\sqrt{u^{2}-4 h^{2}}}{2 h}
$$

is the Zhukovsky variable parameterizing the single cut of $\mathbf{P}$ functions on the defining Riemann sheet. In addition from the analytical structure of $\nu_{i}(u)$ functions on the defining Riemann sheet it follows, that the following combinations of functions

$$
\begin{aligned}
& \nu_{i}(u)+\tilde{\nu}_{i}(u)=\nu_{i}(u)+\sigma \nu_{i}^{[2]}(u), \\
& \frac{\nu_{i}(u)-\tilde{\nu}_{i}(u)}{\sqrt{u^{2}-4 h^{2}}}=\frac{\nu_{i}(u)-\sigma \nu_{i}^{[2]}(u)}{\sqrt{u^{2}-4 h^{2}}}
\end{aligned}
$$

are free of cuts on the whole real axis. Similar to $[64,65]$ we will parametrize the $\mathbf{P}(u)$ functions entering the solution as

$$
\begin{aligned}
& \mathbf{P}_{1}=(x h)^{-L} \mathbf{p}_{1}=(x h)^{-L}\left(1+\sum_{k=1}^{\infty} \sum_{l=0}^{\infty} c_{1, k}^{(l)} \frac{h^{2 l+k}}{x^{k}}\right), \\
& \mathbf{P}_{2}=(x h)^{-L} \mathbf{p}_{2}=(x h)^{-L}\left(\frac{h}{x}+\sum_{k=2}^{\infty} \sum_{l=0}^{\infty} c_{2, k}^{(l)} \frac{h^{2 l+k}}{x^{k}}\right),
\end{aligned}
$$

\footnotetext{
${ }^{1}$ See [65] for details.
} 


$$
\begin{aligned}
& \mathbf{P}_{0}=(x h)^{-L} \mathbf{p}_{0}=(x h)^{-L}\left(\sum_{l=0}^{\infty} A_{0}^{(l)} h^{2 l} u^{L}+\sum_{j=0}^{L-1} \sum_{l=0}^{\infty} m_{j}^{(l)} h^{2 l} u^{j}+\sum_{k=1}^{\infty} \sum_{l=0}^{\infty} c_{0, k}^{(l)} \frac{h^{2 l+k}}{x^{k}}\right) \\
& \mathbf{P}_{3}=(x h)^{-L} \mathbf{p}_{3}=(x h)^{-L}\left(\sum_{l=0}^{\infty} A_{3}^{(l)} h^{2 l} u^{2 L+1}+\sum_{j=0}^{2 L} \sum_{l=0}^{\infty} k_{j}^{(l)} h^{2 l} u^{j}+\sum_{k=1}^{\infty} \sum_{l=0}^{\infty} c_{3, k}^{(l)} \frac{h^{2 l+k}}{x^{k}}\right) .
\end{aligned}
$$

where we have accounted for correct polynomial asymptotic of $\mathbf{P}$ functions at large values of spectral parameter $u$ (2.15). We may always assume that on the defining or first Riemann sheet $|x(u)|>1$ and thus the above expansions are justified. Due to gauge symmetry of QSC equations ${ }^{2}$

$$
\nu_{i} \rightarrow R_{i}^{j} \nu_{j}, \quad \mathbf{P}_{i j} \rightarrow R_{i}^{i^{\prime}} \mathbf{P}_{i^{\prime} j^{\prime}} R_{j}^{j^{\prime}}
$$

where $R$ is any $4 \times 4$ constant matrix satisfying $R^{t} \chi R=\chi$ the coefficients $m_{j}^{(l)}, k_{j}^{(l)}$ in the above parametrization at twist $L=1$ are left undetermined. The coefficients $A_{0}^{(l)}$, $A_{3}^{(l)}$ and $c_{i, k}^{(l)}$ are some functions of spin $S$ only, otherwise they are just constants. Here we have also used the mentioned gauge freedom to set $A_{1}=1$ and $A_{2}=h^{2}$. Since $x$ tends to $x_{+}(u)=\frac{u+i \sqrt{4 h^{2}-u^{2}}}{2 h}$ and $x_{-}(u)=1 / x_{+}(u)=\frac{u-i \sqrt{4 h^{2}-u^{2}}}{2 h}$ on the upper and lower bank of the cut, correspondingly, the values of the functions $\mathbf{P}_{a}(u)$ on the two banks are related as

$$
\mathbf{P}_{a}(u-i 0)=\left.\mathbf{P}_{a}(u+i 0)\right|_{x_{+}(u) \rightarrow 1 / x_{+}(u)} .
$$

Therefore, in a sufficiently small vicinity ${ }^{3}$ of the cut on the second sheet of $u$-plane (the inner vicinity of unit circle in $x$-plane) we have

$$
\tilde{\mathbf{P}}_{a}=\left.\mathbf{P}_{a}\right|_{x \rightarrow 1 / x}=\left(\frac{x}{h}\right)^{L} \tilde{\mathbf{p}}_{a}, \quad \tilde{\mathbf{p}}_{a}=\left.\mathbf{p}_{a}\right|_{x \rightarrow 1 / x} .
$$

Next, the expansion of $\nu_{i}(u)$ functions in terms of QSC coupling constant $h$ is given by

$$
\nu_{i}(u)=\sum_{l=0}^{\infty} h^{2 l-L} \nu_{i}^{(l)}(u) .
$$

\subsection{Leading order}

From now on we consider the case of $L=1$ operators. First, from eqs. (2.15) and (2.14) at large values of spectral parameter $u$ we get

$$
A_{0}^{(0)}=\sigma(2 S+1)
$$

Next, we take LO approximation of the first Baxter equation (3.1). The solution of the latter, as described in detail in the next section, is given by

$$
\nu_{1}^{(0)}(u)=\alpha Q^{[-1]}(S, u)
$$

\footnotetext{
${ }^{2}$ See for details [65].

${ }^{3}$ The vicinity is such that the substitution $x \rightarrow 1 / x$ retains the convergence of $(3.11)-(3.14)$.
} 
where $Q(S, u)$ is LO Baxter polynomial defined in eq. (4.8) and $\alpha$ is some spin $S$ dependent constant to be determined later. In general for the details of the solutions of Baxter equations (3.1)-(3.2) we refer the reader to next section. Here we will just use the results obtained there. Next, from equation (3.3) we determine the expression for $\nu_{3}^{(0)}(u)$ and substitute it in the equation (3.5). Expanding the latter at $u=0$ up to $\mathcal{O}\left(u^{2}\right)$ we get the expression $^{4}$ for constant $\alpha$ :

$$
\frac{1}{\alpha^{2}}=-4 i B_{1}(S), \quad B_{1}(S)=H_{1}(S)-H_{-1}(S) .
$$

Also from the requirement of absence of poles in combinations (3.10) for $\nu_{1}^{(0)}$ we may determine the value of $\sigma=(-1)^{S}$. Knowing the expression for $\nu_{1}^{(0)}(u)$ we may determine $\nu_{2}^{(0)}$ by solving second Baxter equation (3.2):

$$
\begin{aligned}
\nu_{2}^{(0)}= & \sigma \frac{\alpha}{2}\left[-\frac{1}{4} A_{3}^{(0)}\left(\frac{3(S+2)(S+1)}{2 S+3} Q^{[-1]}(S+2, u)-2 \frac{3 S^{2}+3 S+1}{2 S+1} Q^{[-1]}(S, u)\right.\right. \\
& \left.+\frac{3 S(S-1)}{2 S-1} Q^{[-1]}(S-2, u)\right)+\frac{i}{2} k_{2}^{(0)}\left[Q^{[-1]}(S+1, u)-\delta_{S \neq 0} Q^{[-1]}(S-1, u)\right] \\
& \left.+k_{1}^{(0)} \frac{1}{2 S+1} Q^{[-1]}(S, u)\right] .
\end{aligned}
$$

Next, from equation (3.6) expanded at $u=0$ up to $\mathcal{O}(u)$ we get the value of $A_{3}^{(0)}$ constant:

$$
A_{3}^{(0)}=-\frac{4}{3}(2 S+3)(2 S-1) B_{1}(S) .
$$

In addition, from the same expansion we get the following values of coefficients

$$
k_{2}^{(0)}=0, \quad c_{1,1}^{(0)}=0 .
$$

Note, that if we account for $\delta_{S, 0}$ term in the $\nu_{2}^{(0)}$ solution then the value of $k_{2}^{(0)}$ coefficient turns out to be fixed. So, the analytical continuation in spin $S$ allows us to fix extra gauge freedom.

\subsection{Next-to-leading order}

Before starting actual NLO calculation it makes sense to determine as many required constants as possible with the information on LO solutions we already have. Performing small $u$ expansion of (3.6) up to $\mathcal{O}\left(u^{3}\right)$ we get

$$
c_{1,2}^{(0)}=4 B_{1}(S)-B_{1}(S)^{2}-2 B_{2}(S) .
$$

Next, equation (3.7) expanded at $u=0$ up to $\mathcal{O}(1)$ terms gives us the value of $c_{0,1}^{(0)}$ coefficient:

$$
c_{0,1}^{(0)}=\frac{\sigma k_{1}^{(0)}}{2(1+2 S)}-1-\frac{i \sigma S(1+S)}{3(1+2 S) \alpha^{2}} .
$$

\footnotetext{
${ }^{4}$ See appendix B for definition of $B$-sums.
} 
Substituting the expression for $\nu_{4}^{(0)}$ from (3.4) into equation (3.8) and expanding the latter at small $u$ up to $\mathcal{O}(1)$ terms we get the value of $c_{3,1}^{(0)}$ coefficient:

$$
c_{3,1}^{(0)}=\frac{\sigma\left(2 S(1+S)+3 i \alpha^{2} k_{1}^{(0)}\right)\left(12 i(1+2 S) \alpha^{2}-\sigma\left(2 S(1+S)+3 i \alpha^{2} k_{1}^{(0)}\right)\right)}{36(1+2 S)^{2} \alpha^{4}} .
$$

Finally, the expansion of (3.5) at $u=0$ up to $\mathcal{O}\left(u^{4}\right)$ terms gives us the values of $c_{2,2}^{(0)}$ and $c_{2,3}^{(0)}$ coefficients:

$$
c_{2,2}^{(0)}=0, \quad c_{2,3}^{(0)}=4 i \alpha^{2}\left(B_{1}(S) B_{2}(S)+B_{3}(S)\right) .
$$

Now, we are ready to proceed with the solution of NLO Baxter equations. As is explained in the next section the solution of the first Baxter equation (3.1) at NLO is given by

$$
\begin{aligned}
\nu_{1}^{(1)}(u)= & \frac{\alpha}{2}\left(\sigma A_{0}^{(1)}-2(2 S-1) B_{1}(S)\right)\left\{Q ^ { [ - 1 ] } ( S , u ) \left(\gamma_{E}+\log 2-i \eta_{1}(u)\right.\right. \\
& \left.\left.-H_{1}(S)+i \pi \operatorname{coth}(\pi u)\right)+\sum_{k=1}^{S} \frac{1+(-1)^{k}}{k} Q^{[-1]}(S-k, u)\right\} \\
& -\alpha \sum_{k=1}^{S} \frac{1+(-1)^{k}}{k}\left(B_{1}(S)+B_{1}(S-k)\right) Q^{[-1]}(S-k, u) \\
& +\phi_{1,0}^{\text {per }} Q^{[-1]}(S, u)+\phi_{1,1}^{\text {per }} \mathcal{P}_{1}(u) Q^{[-1]}(S, u) .
\end{aligned}
$$

The absence of poles in combinations (3.10) for $\nu_{1}^{(1)}$ allows us to determine the values of coefficients $A_{0}^{(1)}$ and $\phi_{1,1}^{\text {per }}$ :

$$
A_{0}^{(1)}=2 \sigma(3+2 S) B_{1}(S), \quad \phi_{1,1}^{\mathrm{per}}=-2 i \alpha B_{1}(S) .
$$

Next, the expansions of (3.5) at $u=0$ up to $\mathcal{O}\left(u^{2}\right)$ terms fixes the value of $\phi_{1,0}^{\text {per }}$ :

$$
\phi_{1,0}^{\mathrm{per}}=\alpha\left\{\frac{4}{3} B_{1}(S)^{2}+B_{2}(S)+\frac{3 B_{3}(S)+2 H_{3}(S)-2 H_{-3}(S)}{3 B_{1}(S)}-2 B_{1}(S)(1+2 \log 2)\right\} .
$$

Now we are ready to solve the second Baxter equation at NLO (3.2). The details of the solution could be found in the next section. In terms of $q_{2}^{(1)}(u)(4.45)$ the expression for $\nu_{2}^{(1)}(u)$ is then given by

$$
\nu_{2}^{(1)}(u)=q_{2}^{(1)}(u-i / 2)
$$

Requiring the absence of poles in combinations (3.10) for $\nu_{2}^{(1)}$ allows us to fix coefficients $\phi_{2,0}^{\text {per }}$ and $\phi_{2,0}^{\text {anti. }}$

$$
\phi_{2,0}^{\text {per }}=0, \quad \phi_{2,0}^{\text {anti }}=4 i \alpha B_{1}(S) .
$$


Finally, expanding equation (3.6) at $u=0$ up to $\mathcal{O}(1)$ terms gives us the value of $A_{3}^{(1)}$ coefficient:

$$
\begin{aligned}
A_{3}^{(1)}= & -\frac{16}{3}(2 S-1)(2 S+3)\left(3 \bar{H}_{-2,-1}-2 \bar{H}_{-2, i}-\bar{H}_{-2,1}-\bar{H}_{-1,-2}+2 \bar{H}_{-1,2 i}-\bar{H}_{-1,2}\right. \\
& -6 \bar{H}_{i,-2}+12 \bar{H}_{i, 2 i}-6 \bar{H}_{i, 2}-6 \bar{H}_{2 i,-1}+4 \bar{H}_{2 i, i}+2 \bar{H}_{2 i, 1}-\bar{H}_{1,-2}+2 \bar{H}_{1,2 i} \\
& -\bar{H}_{1,2}+3 \bar{H}_{2,-1}-2 \bar{H}_{2, i}-\bar{H}_{2,1}+2 \bar{H}_{-1, i,-1}-2 \bar{H}_{-1, i, 1}+8 \bar{H}_{i,-1,-1} \\
& -12 \bar{H}_{i,-1, i}+4 \bar{H}_{i,-1,1}-16 \bar{H}_{i, i,-1}+16 \bar{H}_{i, i, i}+4 \bar{H}_{i, 1,-1}-4 \bar{H}_{i, 1, i}+2 \bar{H}_{1, i,-1} \\
& \left.-2 \bar{H}_{1, i, 1}-\frac{1}{2} B_{1} \zeta_{2}\right)-\frac{4}{3}\left(5+20 S+4 S^{2}\right) B_{1}^{2} .
\end{aligned}
$$

where $\bar{H}_{a, \ldots}=H_{a, \ldots}(2 S)$ is defined in (5.4). The reason behind the appearance of sums different from harmonic is related to the fact that solutions of Baxter equations at NLO contain Baxter polynomials $Q(S, u)$ under different summation signs with various weights. For example, in the case of homogeneous solution of second Baxter equation $\mathcal{Z}(S, u)$ entering the expression for $q_{2}^{(1)}(u)(4.45)$ its expansion at $u=i / 2$ is given by

$$
\mathcal{Z}\left(S, u+\frac{i}{2}\right)=-i(-1)^{S}\left[S_{-1}(S)+\ln 2\right]+u(-1)^{S}\left[-B_{1}(S) \ln 2+V(S)+\zeta_{2} / 2\right]+\mathcal{O}\left(u^{2}\right)
$$

where $V(S)=\sum_{k=1}^{S} \frac{1+(-1)^{S+k}}{k+S} B_{1}(k-1)$ could be further rewritten in terms of generalized harmonic sums (5.4). Also, the necessity of the argument $2 S$ could be promptly realized after examining the denominators of the rational numbers entering the results for large enough $S$ and observing the appearance of prime numbers in the interval $[S+1,2 S)$ among their factors.

We would like to mention, that in the simplification of the coefficients expressions the use of HarmonicSums mathematica package [88-94] was helpful.

\section{Solution of Baxter equations with Mellin transform}

In the previous section we have seen that the most complicated part of the QSC solution is the solution of two inhomogeneous Baxter equations at each perturbation order. To solve these second order finite difference equations we will employ Mellin transform technique to convert them to ordinary differential equations. The latter was originally applied to the solution of Lipatov's reggeon spin chain [95] by Faddeev and Korchemsky in [96]. Later this technique was used to solve asymptotic Baxter equation in $\mathcal{N}=4 \mathrm{SYM}$ up to three and four loops [97, 98]. In order to iteratively search for the perturbative solution of equations (3.1)-(3.8), we expand eqs. (3.1), (3.2) up to $h^{k}$ and obtain the inhomogeneous equations for $q_{1,2}^{(k)}=\left(\nu_{1,2}^{(k)}\right)^{[1]}$ in the following form

$$
\begin{aligned}
& (u+i / 2) q_{1}^{(k)}(u+i)-i(2 S+1) q_{1}^{(k)}(u)-(u-i / 2) q_{1}^{(k)}(u-i)=V_{1}^{(k)} \\
& (u+i / 2) q_{2}^{(k)}(u+i)+i(2 S+1) q_{2}^{(k)}(u)-(u-i / 2) q_{2}^{(k)}(u-i)=V_{2}^{(k)}
\end{aligned}
$$


Here $V_{1}^{(k)}$ depends on $q_{1,2}^{(l)}$ with $l<k$, and $V_{2}^{(k)}$ depends in addition on $q_{1}^{(k)}$. Applying Mellin transformation ${ }^{5}$ to eqs. (4.1) and (4.2), we get

$$
\begin{aligned}
(\bar{z}-z) \partial_{z} \Psi_{1}^{(k)}(z)+2 S \Psi_{1}^{(k)}(z) & =i \widetilde{V}_{1}^{(k)}(z), \\
(\bar{z}-z) \partial_{z} \Psi_{2}^{(k)}(z)-2(S+1) \Psi_{2}^{(k)}(z) & =i \widetilde{V}_{2}^{(k)}(z),
\end{aligned}
$$

where $\Psi_{1,2}^{(k)}=\mathcal{M}^{-1}\left[q_{1,2}^{(k)}\right]$ and $\widetilde{V}_{1,2}^{(k)}=\mathcal{M}^{-1}\left[V_{1,2}^{(k)}\right]$. The integration of equations (4.3) and (4.4) is straightforward and is given by

$$
\begin{aligned}
& \Psi_{1}^{(k)}(z)=i(\bar{z}-z)^{S} \int(\bar{z}-z)^{-S-1} \widetilde{V}_{1}^{(k)}(z) d z \\
& \Psi_{2}^{(k)}(z)=i(\bar{z}-z)^{-S-1} \int(\bar{z}-z)^{S} \widetilde{V}_{2}^{(k)}(z) d z .
\end{aligned}
$$

Note that, when passing to the Mellin space, we silently assumed that $\Psi_{1,2}^{(k)}(z)$ are finite at $z=0$ and $z=1$. In general it might be not so due to the appearing logarithms of $z$ and $\bar{z}$. Therefore, the approach based on Mellin transformation, should be used with great care, in particular the results obtained within this approach should be transformed back to $u$-space and directly checked against the equations (4.1) and (4.2). These complications may be viewed as disadvantages of the Mellin-space approach. Nevertheless, we find it advantageous to use Mellin transformation technique, at least, up to the next-to-leading order considered in this paper.

\subsection{Homogeneous solution}

The solution of homogeneous first Baxter equation (4.3) in Mellin space is easy and is given by ${ }^{6}$

$$
\Psi_{1}^{(0)}=(\bar{z}-z)^{S}
$$

Its Mellin transform to spectral parameter $u$-space is given by

$$
\begin{aligned}
Q(S, u) & ={ }_{2} F_{1}\left(-S, \frac{1}{2}+i u ; 1 ; 2\right) \\
& =\frac{(-1)^{S} \Gamma\left(\frac{1}{2}+i u\right)}{S ! \Gamma\left(\frac{1}{2}+i u-S\right)}{ }_{2} F_{1}\left(-S, \frac{1}{2}+i u ; \frac{1}{2}+i u-S ;-1\right) .
\end{aligned}
$$

Moreover, $\Phi_{Q}^{\mathrm{per}}(u) Q(S, u)$ and $\Phi_{Q}^{\mathrm{anti}}(u) Q(S, u)$, where $\Phi_{Q}^{\mathrm{per}}(u)$ and $\Phi_{Q}^{\text {anti }}(u)$ are arbitrary periodic and anti-periodic functions of spectral parameter $u$, are also solutions of homogeneous first (4.1) and second (4.2) Baxter equations correspondingly.

To find second solutions of homogeneous Baxter equations let us consider second Baxter equation (4.2). Making the ansatz $q_{2}(S, u)=Q(S, u) b^{[1]}(S, u)$ similar to $[64,65]$ the homogeneous Baxter equation (4.2) could be rewritten as

$$
\nabla_{+}\left(u Q^{[1]} Q^{[-1]} \nabla_{-} b\right)=0,
$$

\footnotetext{
${ }^{5}$ See appendix A for more details and notation.

${ }^{6}$ The arbitrary constant in front of solution is dropped.
} 
where $\nabla_{+} f=f-f^{[2]}$ and $\nabla_{-} f=f+f^{[2]}$. From equation (4.9) it follows then, that ${ }^{7}$

$$
b(u)+b(u+i)=\frac{1}{u Q^{[1]} Q^{[-1]}} .
$$

To solve this difference equation we will use the empirically guessed identity

$$
\begin{aligned}
\frac{1}{u Q^{[1]} Q^{[-1]}}= & \frac{(-1)^{S}}{u}+i(-1)^{S} \sum_{k=0}^{\left\lfloor\frac{S-1}{2}\right\rfloor} \frac{1}{S-k} \\
& \times\left(\frac{Q\left(S-1-2 k, u-\frac{i}{2}\right)}{Q\left(S, u-\frac{i}{2}\right)}+\frac{Q\left(S-1-2 k, u+\frac{i}{2}\right)}{Q\left(S, u+\frac{i}{2}\right)}\right) .
\end{aligned}
$$

Then we see, that

$$
b(u)+b(u+i)=\frac{(-1)^{S}}{u}+\left[i(-1)^{S} \sum_{k=0}^{\left\lfloor\frac{S-1}{2}\right\rfloor} \frac{1}{S-k} \frac{Q\left(S-1-2 k, u-\frac{i}{2}\right)}{Q\left(S, u-\frac{i}{2}\right)}+(u \rightarrow u+i)\right] .
$$

Introducing Hurwitz function $\eta_{-1}(u)$ defined as

$$
\eta_{-1}(u)=\sum_{n=0}^{\infty} \frac{(-1)^{n}}{u+i n}=\frac{i}{2}\left(\psi\left(-i \frac{u}{2}\right)-\psi\left(-i \frac{u+i}{2}\right)\right)
$$

where $\psi(u)$ is polygamma function and noting, that

$$
\eta_{-1}(u)+\eta_{-1}(u+i)=\frac{1}{u}
$$

we see that the solution for $b(u)$ function is given by

$$
b(u)=(-1)^{S} \eta_{-1}(u)+i(-1)^{S} \sum_{k=0}^{\left\lfloor\frac{S-1}{2}\right\rfloor} \frac{1}{S-k} \frac{Q\left(S-1-2 k, u-\frac{i}{2}\right)}{Q\left(S, u-\frac{i}{2}\right)} .
$$

Finally the expression for second solution with polynomial asymptotic, which we will denote by $\mathcal{Z}(S, u)$ is given by ${ }^{8}$

$$
\mathcal{Z}(S, u)=i \sigma \sum_{k=0}^{\left\lfloor\frac{S-1}{2}\right\rfloor} \frac{1}{S-k} Q(S-1-2 k, u)+\sigma \eta_{-1}(u+i / 2) Q(S, u) .
$$

Once this solution is found, one can check directly that it satisfies the homogeneous part of eq. (4.2), using the generating function found in appendix B.

\footnotetext{
${ }^{7}$ Of course, $b(u)$ is defined up to arbitrary multiplicative periodic and additive anti-periodic constants which is taken into account in eqs. (4.17), (4.18).

${ }^{8}$ For a rigorous proof that $\mathcal{Z}(S, u)$ is a solution see appendix B.
} 
The general solutions of first and second homogeneous Baxter equations are then given by

$$
\begin{aligned}
& q_{1}^{\text {hom }}(S, u)=\Phi_{1, \text { per }} Q(S, u)+\Phi_{1, \text { anti }} \mathcal{Z}(S, u), \\
& q_{2}^{\text {hom }}(S, u)=\Phi_{2, \text { anti }} Q(S, u)+\Phi_{2, \text { per }} \mathcal{Z}(S, u),
\end{aligned}
$$

where $\Phi_{i, \text { per }}$ and $\Phi_{i \text {,per }}$ are arbitrary periodic and anti-periodic functions in spectral parameter $u$. Otherwise they are arbitrary functions of spin $S$ to be determined from consistency conditions as described in previous section. We will parametrize their $u$ dependence similar to $[64,65]$ with the basis of periodic and anti-periodic combinations of Hurwitz functions defined as

$$
\mathcal{P}_{k}(u)=\eta_{k}(u)+\eta_{k}(i-u)=\operatorname{sgn}(k) \mathcal{P}_{k}(u+i), \quad k \neq 0 \in \mathbb{Z},
$$

where

$$
\eta_{a}(u)=\sum_{k=0}^{\infty} \frac{(\operatorname{sgn}(a))^{k}}{(u+i k)^{|a|}},
$$

and bar over $\eta$ denotes complex conjugation. Note that $\mathcal{P}_{k}(u)$ can be expressed via elementary functions:

$$
\mathcal{P}_{k}(u)=\frac{\left(-\partial_{u}\right)^{|k|-1}}{(|k|-1) !}\left\{\begin{array}{ll}
\pi \operatorname{coth}(\pi u) & k>0 \\
\pi / \cosh (\pi u) & k<0
\end{array} .\right.
$$

Then the functions $\Phi_{a}^{\mathrm{per}}$ and $\Phi_{a}^{\mathrm{anti}}$ are written as

$$
\Phi_{a}^{\mathrm{per}}(u)=\phi_{a, 0}^{\mathrm{per}}+\sum_{j=1}^{\Lambda} \phi_{a, j}^{\mathrm{per}} \mathcal{P}_{j}(u), \quad \Phi_{a}^{\mathrm{anti}}(u)=\sum_{j=1}^{\Lambda} \phi_{a, j}^{\mathrm{anti}} \mathcal{P}_{-j}(u),
$$

where $\Lambda$ is a cutoff dependent on the order of perturbation theory.

\subsection{Inhomogeneous solution}

Let us now proceed with the solution of inhomogeneous Baxter equations.

\subsubsection{LO}

At leading order $V_{1}^{(0)}=0$ and LO order solution of the first Baxter equation is given by the solution of homogeneous equation:

$$
\Psi_{1}^{(0)}=\alpha(\bar{z}-z)^{S}, \quad q_{1}^{(0)}(u)=\alpha Q(S, u)
$$

with $\alpha$ given by equation (3.20). The leading order second Baxter equation is already inhomogeneous with $V_{2}^{(0)}$, after substitution of the anzats for $\mathbf{P}$-functions, given by

$$
\begin{aligned}
& V_{2}^{(0)}=i \sigma\left[A_{3}^{(0)}\left(3 u^{2}-\frac{1}{4}\right)+2 k_{2}^{(0)} u+k_{1}^{(0)}\right] q_{1}^{(0)}, \\
& \widetilde{V}_{2}(z)=i \sigma\left[A_{3}^{(0)}\left(3 \hat{u}^{2}-\frac{1}{4}\right)+2 k_{2}^{(0)} \hat{u}+k_{1}^{(0)}\right] \Psi_{1}^{(0)},
\end{aligned}
$$


and the particular solution for $\Psi_{2}^{(0)}$ is given by (4.6):

$$
\begin{aligned}
\Psi_{2}^{(0)}(z) & =-\sigma(\bar{z}-z)^{-S-1} \int(\bar{z}-z)^{S}\left[A_{3}^{(0)}\left(3 \hat{u}^{2}-\frac{1}{4}\right)+2 k_{2}^{(0)} \hat{u}+k_{1}^{(0)}\right] \Psi_{1}^{(0)} d z \\
& =-\sigma \alpha(\bar{z}-z)^{-S-1} \int(\bar{z}-z)^{S}\left[A_{3}^{(0)}\left(3 \hat{u}^{2}-\frac{1}{4}\right)+2 k_{2}^{(0)} \hat{u}+k_{1}^{(0)}\right](\bar{z}-z)^{S} d z
\end{aligned}
$$

Acting by $\hat{u}$ operator (see eq. (A.7)), we obtain

$$
\begin{aligned}
\Psi_{2}^{(0)}(z)= & \sigma \frac{1}{2} \alpha(\bar{z}-z)^{-S-1} \int d(\bar{z}-z)(\bar{z}-z)^{S}\left[-\frac{1}{4} A_{3}^{(0)}\left(3(S+2)(S+1)(\bar{z}-z)^{S+2}\right.\right. \\
& \left.-2\left[3 S^{2}+3 S+1\right](\bar{z}-z)^{S}+3 S(S-1)(\bar{z}-z)^{S-2}\right) \\
& \left.+i k_{2}^{(0)}\left[(S+1)(\bar{z}-z)^{S+1}-S(\bar{z}-z)^{S-1}\right]+k_{1}^{(0)}(\bar{z}-z)^{S}\right] \\
= & \sigma \frac{1}{2} \alpha\left[C(\bar{z}-z)^{-S-1}-\frac{1}{4} A_{3}^{(0)}\left(3(S+2)(S+1) \frac{(\bar{z}-z)^{S+2}}{2 S+3}\right.\right. \\
& \left.-2\left[3 S^{2}+3 S+1\right] \frac{(\bar{z}-z)^{S}}{2 S+1}+3 S(S-1) \frac{(\bar{z}-z)^{S-2}}{2 S-1}\right) \\
& \left.+\frac{i}{2} k_{2}^{(0)}\left[(\bar{z}-z)^{S+1}-(\bar{z}-z)^{S-1}\right]+k_{1}^{(0)} \frac{(\bar{z}-z)^{S}}{2 S+1}\right]
\end{aligned}
$$

The requirement that $\Psi_{2}^{(0)}(z)$ is polynomial fixes constant $C=\frac{i}{2} k_{2}^{(0)} \delta_{S, 0}$ and we finally get ${ }^{9}$

$$
\begin{aligned}
q_{2}^{(0)}= & \sigma \frac{\alpha}{2}\left[-\frac{1}{4} A_{3}^{(0)}\left(\frac{3(S+2)(S+1)}{2 S+3} Q(S+2, u)-2 \frac{3 S^{2}+3 S+1}{2 S+1} Q(S, u)\right.\right. \\
& \left.+\frac{3 S(S-1)}{2 S-1} Q(S-2, u)\right)+\frac{i}{2} k_{2}^{(0)}\left[Q(S+1, u)-\delta_{S \neq 0} Q(S-1, u)\right] \\
& \left.+k_{1}^{(0)} \frac{1}{2 S+1} Q(S, u)\right] .
\end{aligned}
$$

\subsubsection{NLO}

At NLO the inhomogeneous part of the first Baxter equation after substitution of the anzats for $\mathbf{P}$-functions is given by

$$
\begin{aligned}
V_{1}^{(1)}= & \frac{4 i \sigma q_{2}^{(0)}(u)}{1+4 u^{2}}+\left(c_{1,1}^{(0)}+\frac{2}{2 u+i}\right) q_{1}^{(0)}(u+i)-\left(c_{1,1}^{(0)}+\frac{2}{2 u-i}\right) q_{1}^{(0)}(u-i) \\
& +\sigma\left\{i A_{0}^{(1)}-\frac{4 i}{1+4 u^{2}}\left(c_{0,1}^{(0)}-c_{1,1}^{(0)} m_{0}^{(0)}\right)\right\} q_{1}^{(0)}(u) .
\end{aligned}
$$

To convert it to Mellin space we need the following expressions

$$
\begin{aligned}
\frac{q_{1}^{(0)(u+i)}}{u+i / 2} & =\alpha\left[i \bar{z} \partial_{z} z\right]^{-1}\left[-\frac{\bar{z}}{z}(\bar{z}-z)^{S}+\frac{1}{z}\right]=i \frac{\alpha}{z} \partial_{z}^{-1} z^{-1}\left[(\bar{z}-z)^{S}-\frac{1}{\bar{z}}\right] \\
& =i \frac{\alpha}{z} \int_{0}^{z} \frac{d x}{x}\left[(\bar{x}-x)^{S}-\bar{x}^{-1}\right]=i \frac{\alpha}{z}[\ln \bar{z}+G(S, z)],
\end{aligned}
$$

\footnotetext{
${ }^{9}$ The homogeneous piece at this order is zero as could be seen from the consistency constraints considered in previous section.
} 


$$
\begin{aligned}
\frac{q_{1}^{(0)(u-i)}}{u-i / 2} & =\alpha\left[i z \partial_{z} \bar{z}\right]^{-1}\left[-\frac{z}{\bar{z}}(\bar{z}-z)^{S}+\frac{\sigma}{\bar{z}}\right]=-i \alpha \bar{z}^{-1} \partial_{\bar{z}}^{-1}\left[\frac{1}{\bar{z}}(\bar{z}-z)^{S}-\frac{\sigma}{z \bar{z}}\right] \\
& =-i \sigma \frac{\alpha}{\bar{z}}[\ln z+G(S, \bar{z})]
\end{aligned}
$$

where

$$
\begin{aligned}
G(S, z) & =\int_{0}^{z} \frac{d x}{x}\left[(\bar{x}-x)^{S}-\bar{x}^{-1}\right]-\ln \bar{z} \\
& =\int_{0}^{z} \frac{d x}{x}\left[(\bar{x}-x)^{S}-1\right]=\partial_{z}^{-1} z^{-1}\left[(\bar{z}-z)^{S}-1\right] .
\end{aligned}
$$

The introduced $G$-function satisfies the following recurrence relation

$$
G(S, z)-G(S-1, z)=-2 \int_{0}^{z} d x(1-2 x)^{S-1}=\frac{1}{S}\left((\bar{z}-z)^{S}-1\right), \quad G(0, z)=0 .
$$

So, we may write it as

$$
G(S, z)=\sum_{j=1}^{S} \frac{1}{j}\left((\bar{z}-z)^{j}-1\right) .
$$

The contribution of the term proportional to $q_{2}^{(0)}(u)$ is determined using partial fractioning

$$
\frac{4 i}{1+4 u^{2}}=\frac{1}{u-i / 2}-\frac{1}{u+i / 2}
$$

so that

$$
\begin{aligned}
\frac{4 i \widetilde{Q(S, u)}}{1+4 u^{2}} & =-i\left[\left(-1+\bar{z} \partial_{z}\right)^{-1} z^{-1}-\left(1+z \partial_{z}\right)^{-1} \bar{z}^{-1}\right](\bar{z}-z)^{S} \\
& =-i\left\{\bar{z}^{-1} G(S, z)+\sigma z^{-1} G(S, \bar{z})+\bar{z}^{-1} \ln z+\sigma z^{-1} \ln \bar{z}+\underline{\left(\bar{z}^{-1}+z^{-1} \sigma\right) B_{1}(S)}\right\} .
\end{aligned}
$$

The underlined term is integration constant chosen in order to get rid of singularities at $z=0$ and $z=1$. Using the above expressions together with the values of constants known at this stage we get

$$
\begin{aligned}
& \frac{\widetilde{V_{1}^{(1)}}(z)}{\alpha}=\frac{i}{2}\left[(2 S-1)(\bar{z}-z)^{S+2}-(2 S+3)(\bar{z}-z)^{S}(\bar{z}-z)(\sigma-1)+(1+\sigma)\right] \frac{B_{1}(S)}{(z \bar{z})} \\
& +\frac{i}{2}(z \bar{z})^{-1}((1+\sigma)(\bar{z}-z)+(1-\sigma)) \delta G(S, z)+i \sigma A_{0}^{(1)}(\bar{z}-z)^{S},
\end{aligned}
$$

where

$$
\delta G(S, z) \equiv G(S, z)-G(S, \bar{z})=\sum_{j=1}^{S} \frac{1-(-1)^{j}}{j}(\bar{z}-z)^{j} .
$$

Rewriting the above expression as

$$
\begin{aligned}
\frac{1}{z \bar{z}} \delta G(S, z) & =H(S, z)-H(S, \bar{z})+\left(\frac{1}{z}-\frac{1}{\bar{z}}\right) B_{1}(S), \\
\frac{\bar{z}-z}{z \bar{z}} \delta G(S, z) & =H(S, z)+H(S, \bar{z})+\left(\frac{1}{z}+\frac{1}{\bar{z}}\right) B_{1}(S),
\end{aligned}
$$


where

$$
H(S, z)=\sum_{j=1}^{S} \frac{1-(-1)^{j}}{j z}\left((\bar{z}-z)^{j}-1\right)=-2 \sum_{k=0}^{S-1}(\bar{z}-z)^{k}\left\{B_{1}(S)-B_{1}(k)\right\},
$$

we may get rid from $z, \bar{z}$ in the denominator and the final expression for $\widetilde{V}_{1}^{(1)}(z)$ takes the form $(w=\bar{z}-z)$ :

$$
\frac{\widetilde{V}_{1}^{(1)}(z)}{\alpha i}=w^{S}\left(\sigma A_{0}^{(1)}-2(2 S-1) B_{1}(S)\right)+2 \sum_{k=1}^{S}\left(1+(-1)^{k}\right)\left\{B_{1}(S)+B_{1}(S-k)\right\} w^{S-k},
$$

Then the particular solution is given by (4.5):

$$
\begin{aligned}
\Psi_{1}^{(1)}(z)= & \frac{\alpha}{2}\left(\sigma A_{0}^{(1)}-2(2 S-1) B_{1}(S)\right) w^{S} \log w \\
& -\alpha \sum_{k=1}^{S} \frac{1+(-1)^{k}}{k} w^{S-k}\left\{B_{1}(S)+B_{1}(S-k)\right\},
\end{aligned}
$$

For the Mellin transformed ${ }^{10}$ to $u$-space particular solution plus homogeneous piece with at maximum first order poles in $u$ as required by QSC analyticity constraints we then get ${ }^{11}$

$$
\begin{aligned}
q_{1}^{(1)}(u)= & \frac{\alpha}{2}\left(\sigma A_{0}^{(1)}-2(2 S-1) B_{1}(S)\right)\left\{Q ( S , u ) \left(\gamma_{E}+\log 2-i \eta_{1}(u+i / 2)\right.\right. \\
& \left.\left.-H_{1}(S)+i \pi \tanh (\pi u)\right)+\sum_{k=1}^{S} \frac{1+(-1)^{k}}{k} Q(S-k, u)\right\} \\
& -\alpha \sum_{k=1}^{S} \frac{1+(-1)^{k}}{k}\left(B_{1}(S)+B_{1}(S-k)\right) Q(S-k, u) \\
& +\phi_{1,0}^{\text {per }} Q(S, u)+\phi_{1,1}^{\text {per }} \mathcal{P}_{1}(u+i / 2) Q(S, u) .
\end{aligned}
$$

The solution of second Baxter equation at NLO goes along the same lines, but the expressions become more cumbersome. So, here we will present only the expression for $q_{2}^{(1)}(u)$ without derivation. The latter including homogeneous piece with at maximum first order poles in $u$ as required by QSC analyticity constraints is given by

$$
\begin{aligned}
& \frac{q_{2}^{(1)}(u)}{\alpha} \\
& =A_{3}^{(1)}\left(\frac{\sigma\left(3 S^{2}+3 S+1\right) Q_{S}}{4(2 S+1)}-\frac{3 \sigma(S-1) S Q_{S-2}}{8(2 S-1)}-\frac{3 \sigma(S+1)(S+2) Q_{S+2}}{8(2 S+3)}\right) \\
& \quad+2(2 S+3) B_{1}(S)\left\{\left(\frac{1}{2\left\lfloor\frac{S+1}{2}\right\rfloor-1}+\sigma\right) Q_{S-2}+\frac{(1-\sigma(2 S-1)) Q_{S}}{2 S+1}\right\}-4 B_{1}(S) G_{3}(S)
\end{aligned}
$$

\footnotetext{
${ }^{10}$ See the details of transformation in appendix C.

${ }^{11}$ Here we have dropped the piece with non-polynomial asymptotic in the particular solution contribution. The latter is defined up to a homogeneous solution and we may use this freedom to ensure that the ansatz for overall solution has correct polynomial asymptotics we are looking for.
} 


$$
\begin{aligned}
& -\frac{k_{1}^{(0)} \sigma(2 S+3) B_{1}(S) Q_{S}}{(2 S+1)^{2}}+\left(\frac{\sigma(2 S-1)(2 S+3)\left(3 S^{2}+3 S+1\right) B_{1}(S)}{3(2 S+1)}-\frac{k_{1}^{(0)} \sigma}{2(2 S+1)}\right) \\
& \times\left\{2 B_{1}(S) G_{6}(S)+G_{4}(S)-\alpha^{-1} \Phi_{1}^{\mathrm{per}}(u) Q_{S}\right\}+\frac{1}{4} i k_{2}^{(1)} \sigma\left(Q_{S+1}-\delta_{S \neq 0} Q_{S-1}\right) \\
& -\frac{1}{2} \sigma(S-1) S(2 S+3) B_{1}(S)\left\{\left(2 B_{1}(S) G_{6}(S-2)+G_{4}(S-2)-\alpha^{-1} \Phi_{1}^{\mathrm{per}}(u) Q_{S-2}\right\}\right. \\
& -\frac{1}{2} \sigma(S+1)(S+2)(2 S-1) B_{1}(S)\left\{2 B_{1}(S) G_{6}(S+2)+G_{4}(S+2)-\alpha^{-1} \Phi_{1}^{\mathrm{per}}(u) Q_{S+2}\right\} \\
& +\sigma(2 S-1)(2 S+3) B_{1}(S)^{2}\left\{\frac{\left(6 S^{3}-3 S^{2}-11 S-3\right) Q_{S}}{3(2 S+1)^{2}}-\frac{\left(2 S^{3}-3 S^{2}-23 S-22\right) Q_{S+2}}{2(2 S+3)^{2}}\right. \\
& \left.-\frac{(S-1) S(2 S+3) Q_{S-2}}{2(2 S-1)^{2}}\right\}+\frac{k_{1}^{(1)} \sigma Q_{S}}{2(2 S+1)}+\alpha^{-1} \phi_{2,0}^{\text {per }} \mathcal{Z}_{S}+\alpha^{-1} \phi_{2,0}^{\text {anti }} \mathcal{P}_{-1}\left(u+\frac{i}{2}\right) Q_{S},
\end{aligned}
$$

where $Q_{S} \equiv Q_{S}(u) \equiv Q(S, u), \mathcal{Z}_{S} \equiv \mathcal{Z}(S, u)$ and the following functions were introduced:

$$
\begin{aligned}
G_{3}(S) \equiv G_{3}(S, u)= & \sum_{k=S+1}^{2 S+1} \frac{\left(1-(-1)^{k}\right) Q_{k-S-1}(u)}{k} \\
G_{4}(S) \equiv G_{4}(S, u)= & -\sum_{k=1}^{S} \frac{\left(1+(-1)^{k}\right) Q_{S-k}(u)}{k}\left(B_{1}(S)-B_{1}(S-k)\right) \\
G_{6}(S) \equiv G_{6}(S, u)= & -\sum_{k=1}^{S} \frac{\left(1+(-1)^{k}\right) Q_{S-k}(u)}{k} \\
& -2 Q_{S}(u)\left\{\gamma_{E}+\log 2-H_{1}(S)-i \eta_{1}(u+i / 2)+i \pi \tanh (\pi u)\right\}
\end{aligned}
$$

\section{Anomalous dimensions}

The expressions for the anomalous dimensions could be easily obtained from $A_{3}^{(0,1)}$ with the help of (2.15). This way, up to four loops we got the following results for anomalous dimensions of twist 1 operators

$$
\gamma(S)=\gamma^{(0)}(S) h^{2}+\gamma^{(1)}(S) h^{4}+\ldots
$$

where

$$
\begin{aligned}
\gamma^{(0)}(S)= & 4\left(H_{1}-H_{-1}\right) \\
\gamma^{(1)}(S)= & 16\left\{3 \bar{H}_{-2,-1}-2 \bar{H}_{-2, i}-\bar{H}_{-2,1}-\bar{H}_{-1,-2}+2 \bar{H}_{-1,2 i}-\bar{H}_{-1,2}-6 \bar{H}_{i,-2}\right. \\
& +12 \bar{H}_{i, 2 i}-6 \bar{H}_{i, 2}-6 \bar{H}_{2 i,-1}+4 \bar{H}_{2 i, i}+2 \bar{H}_{2 i, 1}-\bar{H}_{1,-2}+2 \bar{H}_{1,2 i}-\bar{H}_{1,2} \\
& +3 \bar{H}_{2,-1}-2 \bar{H}_{2, i}-\bar{H}_{2,1}+2 \bar{H}_{-1, i,-1}-2 \bar{H}_{-1, i, 1}+8 \bar{H}_{i,-1,-1}-12 \bar{H}_{i,-1, i} \\
& \left.+4 \bar{H}_{i,-1,1}-16 \bar{H}_{i, i,-1}+16 \bar{H}_{i, i, i}+4 \bar{H}_{i, 1,-1}-4 \bar{H}_{i, 1, i}+2 \bar{H}_{1, i,-1}-2 \bar{H}_{1, i, 1}\right\} \\
& +8\left(H_{-1}-H_{1}\right) \zeta_{2},
\end{aligned}
$$


and we introduced new sums

$$
H_{a, b, \ldots}(S)=\sum_{k=1}^{S} \frac{\Re\left[(a /|a|)^{k}\right]}{k^{|a|}} H_{b, \ldots}(k), \quad H_{a, \ldots}=H_{a, \ldots}(S), \quad \bar{H}_{a, \ldots}=H_{a, \ldots}(2 S),
$$

so that for real indexes these sums reduce to ordinary harmonic sums. Note that the conventional harmonic sums of argument $S$ can be expressed via our extended basis (5.4). In particular, we have $\gamma^{(0)}(S)=4\left(H_{1}-H_{-1}\right)=4\left(\bar{H}_{1}+\bar{H}_{-1}-2 \bar{H}_{i}\right)$.

Imaginary indexes correspond to the generalization of the harmonic sums with the fourth root of unity factor $(\exp (i \pi / 2))^{n}$. We would like also to point out the appearance of argument $2 S$ and note, that these new sums could not be further reduced to cyclotomic or other generalized harmonic sums of [90, 91]. From this expression we see, that the maximal transcendentality principle ${ }^{12}[70,71]$ also holds for anomalous dimensions of ABJM theory with the account for finite size corrections. Next, the obtained expression respects shift symmetry [65, 77], that is maximum transcendentality parts $\left(\zeta_{2}\right.$ pieces in our case $)$ of anomalous dimensions of operators with $(L, S)=(1,2 n)$ and $(L, S)=(1,2 n-1), n \in \mathbb{N}^{+}$ are the same. It is also interesting to inspect the large spin $S$ limit of our expressions. In this limit we get

$$
\begin{aligned}
& \gamma^{(0)}(S) \sim 4 \log (S)+4\left(\log 2+\gamma_{E}\right)+\mathcal{O}\left(\frac{1}{S}\right), \\
& \gamma^{(1)}(S) \sim-8 \zeta_{2} \log (S)-8 \zeta_{2}\left(\log 2+\gamma_{E}\right)-12 \zeta_{3}+\mathcal{O}\left(\frac{\log (S)}{S}\right),
\end{aligned}
$$

which is in agreement with [77, 82], where it was shown that wrapping correction scales as $\log S / S$.

Let us now make some comments on the size of the basis of generalized harmonic sums (5.4) at NNLO and higher. At weight $w$ the number of all such sums is given by $4 \cdot 5^{w-1}\left(3 \cdot 4^{w-1}\right.$ if we take into account the real part operator in (5.4)). However, similar to S-sums $[90,102]$ these sums should obey additional relations similar to those following from quasi-shuffle algebra together with differentiation and generalized argument relations for S-sums. Indeed at NLO with weight 3 we expect in total 100 (48) such sums. However, only 29 of them appear in our final answer (5.3). Similarly, at NNLO with $w=5$ in general we should have 2500 (768) sums which are expected to further reduce to several hundreds. Also, in the case of ABJM model compared to $\mathcal{N}=4$ SYM the analyticity properties of anomalous dimensions are fully unstudied at the moment and we can not use for example such notion as Gribov-Lipatov reciprocity $[103,104]$ to further reduce sums basis, see for example [76].

Finally, it is instructive to compare our result with previously known expression [77, 82]:

$$
\begin{aligned}
\gamma^{L=1, S}= & 4 h^{2}\left(H_{1}-H_{-1}\right)-16 h^{4}\left(H_{-3}-H_{3}+H_{-2,-1}-H_{-2,1}+H_{-1,-2}-H_{-1,2}\right. \\
& \left.-H_{1,-2}+H_{1,2}-H_{2,-1}+H_{2,1}+H_{-1,-1,-1}-H_{-1,-1,1}-H_{1,-1,-1}+H_{1,-1,1}\right) \\
& +4 h^{4}\left(H_{1}-H_{-1}\right) \mathcal{W}(1, S)+\mathcal{O}\left(h^{6}\right),
\end{aligned}
$$

\footnotetext{
${ }^{12}$ Similar considerations for the evaluation of Feynman diagrams first appeared in [99]. See also [100, 101].
} 
where

$$
\begin{aligned}
\mathcal{W}(L, S)= & -\frac{i}{2} \sum_{M=1}^{\infty}(-1)^{M} \operatorname{Res}_{q=i M} \frac{4^{L} Q\left(\frac{q-i(M-1)}{2}\right)}{\left(q^{2}+M^{2}\right)^{L} Q\left(\frac{q-i(M+1)}{2}\right) Q\left(\frac{q+i(M-1)}{2}\right) Q\left(\frac{q+i(M+1)}{2}\right)} \\
& \times \sum_{j=0}^{M-1}\left[\frac{Q\left(\frac{q-i(M-1)+2 i j}{2}\right)}{Q\left(\frac{q-i(M-1)}{2}\right)}\right]^{2}\left(\frac{1}{2 j-i q-M}-\frac{1}{2(j+1)-i q-M}\right)
\end{aligned}
$$

and $Q(u)={ }_{2} F_{1}(-S, i u+1 / 2 ; 1 ; 2)$. This expression is still too complex as it involves "complex" hypergeometric functions and takes more time to get expressions for anomalous dimensions compared to our representation in terms of generalized harmonic sums. Moreover the representation in terms of sums makes the study of analytic properties of anomalous dimensions much more simple.

\section{Conclusion}

In this paper we have shown how Mellin space technique could be used to solve multiloop Baxter equations arising from $\mathcal{N}=4 \mathrm{SYM}$ or ABJM quantum spectral curves. As a particular example we have considered anomalous dimensions of twist 1 operators in ABJM theory up to four loop order. The result could be expressed in terms of harmonic sums with imaginary indexes, so that the maximum transcendentality principle holds. The presented Mellin space technique for the solution of multiloop Baxter equations arising within quantum spectral curve method could be extended to higher loops at twist 1. Moreover, it could be also applied to the twist 2 operators. In the latter case we will have an inhomogeneous second order differential equation in Mellin space, which could be further solved using Abel's reduction of order to find second homogeneous solution ${ }^{13}$ and variation of constants to determine particular solution of inhomogeneous equation. However, the main technical difficulty within the presented approach is related to finding Mellin and inverse Mellin transforms of arising functions. Our preliminary results on the solution of multiloop Baxter equations directly in spectral parameter $u$-space for arbitrary spin values show that such a direct approach could be much more effective both for having results at higher loops and twists. So, next we are planning to concentrate on developing corresponding $u$-space techniques.

Finally, we would like to note that the presented techniques could be also applied for solving twisted $\mathcal{N}=4$ and ABJM quantum spectral curves, where $\mathbf{P}$ function will have twisted non-polynomial asymptotic at large spectral parameter values. The latter models are interesting in the connection with the recent progress with the so called fishnet theories [105-112].

\section{Acknowledgments}

This work was supported by RFBR grants \# 17-02-00872, \# 16-02-00943 and contract \# 02.A03.21.0003 from 27.08.2013 with Russian Ministry of Science and Education. The work of R. Lee was supported by the grant of the "Basis" foundation for theoretical physics.

\footnotetext{
${ }^{13}$ The first homogeneous solution is known for arbitrary spin values.
} 


\section{A Mellin transformation}

We define Mellin transformation and its inverse similar to [98], that is

$$
\begin{aligned}
& f(u)=\mathcal{M}[\tilde{f}](u)=\frac{1}{K_{1}(u)} \int_{0}^{1} d z z^{i u-\frac{1}{2}} \bar{z}^{-i u-\frac{1}{2}} \tilde{f}(z), \\
& \tilde{f}(z)=\mathcal{M}^{-1}[f](z)=\frac{1}{2 \pi} \int_{-\infty}^{\infty} d u z^{-i u-\frac{1}{2}} \bar{z}^{i u-\frac{1}{2}} K_{1}(u) f(u),
\end{aligned}
$$

where

$$
K_{1}(u)=\Gamma\left(\frac{1}{2}+i u\right) \Gamma\left(\frac{1}{2}-i u\right)=\frac{\pi}{\cosh (\pi u)},
$$

and $\bar{z}=1-z$. Next, it is easy to see, that the introduced transformations have the following properties

$$
\begin{aligned}
\widetilde{u f(u)}(z) & =\hat{u} \tilde{f}(z)=\frac{i}{2}\left(\bar{z}-z+2 z \bar{z} \partial_{z}\right) \tilde{f}(z), \\
\widetilde{f(u+i)}(z) & =-\frac{\bar{z}}{z} \tilde{f}(z)+\frac{1}{z} f(i / 2)=-\frac{\bar{z}}{z} \tilde{f}(z)+\frac{1}{z} \tilde{f}(0), \\
f \widetilde{(u-i)}(z) & =-\frac{z}{\bar{z}} \tilde{f}(z)+\frac{1}{\bar{z}} f(-i / 2)=-\frac{z}{\bar{z}} \tilde{f}(z)+\frac{1}{\bar{z}} \tilde{f}(1) .
\end{aligned}
$$

Eqs. (A.5) and (A.6) are valid when $f(u)$ does not have singularities in the strip $0<\Im u<1$ and $-1<\Im u<0$, respectively. It is also convenient to introduce the following shorthand notations widely used in the main body of the paper

$$
\begin{aligned}
\hat{u} & =\frac{i}{2}\left(\bar{z}-z+2 z \bar{z} \partial_{z}\right)=\sqrt{z \bar{z}} i \partial_{z} \sqrt{z \bar{z}}, \\
f^{[n]}(u) & =f(u+i n / 2) .
\end{aligned}
$$

The Mellin transform and its inverse convert polynomials into polynomials. Moreover, polynomials of degree $k$ are converted into polynomials with the same degree. That is

$$
\frac{1}{K_{1}(u)} \int_{0}^{1} d z z^{i u-\frac{1}{2}} \bar{z}^{-i u-\frac{1}{2}} z^{k}=\frac{\Gamma\left(\frac{1}{2}+i u+k\right) \Gamma\left(\frac{1}{2}-i u\right)}{K_{1}(u) k !}=\frac{\left(\frac{1}{2}+i u\right)_{k}}{k !}
$$

and

$$
\frac{1}{2 \pi} \int_{-\infty}^{\infty} d u z^{-i u-\frac{1}{2}} \bar{z}^{i u-\frac{1}{2}} K_{1}(u) u^{k}=\hat{u}^{k} 1=\left[\frac{i}{2}\left(\bar{z}-z+2 z \bar{z} \partial_{z}\right)\right]^{k} 1 .
$$

\section{B Generating function and properties of Baxter polynomials}

Let us define the generating function for Baxter polynomials as

$$
W(x, u)=\sum_{S=0}^{\infty} Q(S, u) x^{S} .
$$

In order to obtain the differential equation for $W(x, u)$, we use the relation

$$
\begin{gathered}
-a^{2}{ }_{2} F_{1}(a+1, b ; a+b+1 ;-1)+(a+b-1)(a+b){ }_{2} F_{1}(a-1, b ; a+b-1 ;-1) \\
-(2 b-1)(a+b){ }_{2} F_{1}(a, b ; a+b ;-1)=0 .
\end{gathered}
$$


Putting $a=-S, b=\frac{1}{2}+i u$ we obtain the relation between the Baxter polynomials ${ }^{14}$

$$
S Q(S-1, u)-(S+1) Q(S+1, u)-2 i u Q(S, u)=0 .
$$

Multiplying the above recurrence relation with $x^{S}$ and summing over $S$, we obtain

$$
x \partial_{x} x W(x, u)-\partial_{x} W(x, u)-2 i u W(x, u)=0 .
$$

Solving this differential equation with the boundary condition $W(0, u)=Q(0, u)=1$, we have

$$
W(x, u)=(1-x)^{-\frac{1}{2}+i u}(1+x)^{-\frac{1}{2}-i u}=\frac{e^{-2 i(u-i / 2) \operatorname{arctanh} x}}{1-x},
$$

We will use this generating function $W(x, u)$ for two purposes. First, let us prove that

$$
\mathcal{Z}(S, u)=i \sigma \sum_{k=0}^{\left\lfloor\frac{S-1}{2}\right\rfloor} \frac{1}{S-k} Q(S-1-2 k, u)+\sigma \eta_{-1}(u+i / 2) Q(S, u)
$$

is a solution of the homogeneous part of the second Baxter equation (4.2), i.e., that

$$
(u+i / 2) \mathcal{Z}(S, u+i)+i(2 S+1) \mathcal{Z}(S, u)-(u-i / 2) \mathcal{Z}(S, u-i)=0 .
$$

Substituting (B.6) in the left-hand side, we obtain

$$
\begin{aligned}
(u+i / 2) \mathcal{Z}(u+i) & +i(2 S+1) \mathcal{Z}(u)-(u-i / 2) \mathcal{Z}(u-i)=Q(S, u+i)-Q(S, u-i) \\
& +2 i \sum_{k=0}^{S-1} \frac{1-(-1)^{k+S}}{2 k+1}[(u+i / 2) Q(k, u+i)-(u-i / 2) Q(k, u-i)]
\end{aligned}
$$

We have used here the first Baxter equation to express $Q(S, u)$ and $Q(S-1-2 k, u)$ via $Q(S, u \pm i)$ and $Q(S-1-2 k, u \pm i)$, respectively. We also used the identity $\eta_{-1}(u)+$ $\eta_{-1}(u+i)=u^{-1}$. Now, to prove that the right-hand side of eq. (B.8) is zero, we sum it over $S$ with the weight $x^{S}$. Then we have

$$
\begin{aligned}
\sum_{S=0}^{\infty} x^{S}[\text { r.h.s. of }(\mathrm{B} .8)]= & W(x, u+i)-W(x, u-i) \\
& +2 i \sum_{k=0}^{\infty} \frac{1}{2 k+1}[(u+i / 2) Q(k, u+i)-(u-i / 2) Q(k, u-i)] \\
& \times \sum_{S=k+1}^{\infty} x^{S}\left(1-(-1)^{k+S}\right) \\
= & W(x, u+i)-W(x, u-i) \\
& +\frac{2 i \sqrt{x}}{1-x^{2}} \int_{0}^{x} \frac{d y}{\sqrt{y}}\left[\left(u+\frac{i}{2}\right) W(y, u+i)-\left(u-\frac{i}{2}\right) W(y, u-i)\right] .
\end{aligned}
$$

\footnotetext{
${ }^{14}$ There is another way to find this relation by noting the formal symmetry of the definition (4.8) with respect to the substitution $-S \leftrightarrow 1 / 2+i u$ and applying this substitution to the homogeneous part of Baxter equation (4.1).
} 
Then, using the explicit form of $W(x, u)$, eq. (B.5), we can check that the last expression is zero.

Now, let us derive a closed expression for the derivatives of Baxter polynomials at $u=i / 2$. From (B.5) we have

$$
\left.\partial_{u}^{n} W(x, u)\right|_{u=i / 2}=\frac{(-2 i \operatorname{arctanh} x)^{n}}{1-x} .
$$

The coefficient in front of $x^{S}$ is the $n$-th derivative of $Q(S, u)$ at $u=i / 2$. Using $\frac{1}{1-x}=\sum x^{n}$ we obtain

$$
Q^{(n)}(S, i / 2)=\left.(-2 i)^{n}\left\langle(\operatorname{arctanh} x)^{n}\right\rangle_{S}\right|_{x=1}
$$

where

$$
\langle f(x)\rangle_{S}=\sum_{k=0}^{S} \frac{f^{(k)}(0)}{k !} x^{k}
$$

denotes the Taylor expansion of the function $f(x)$ interrupted at $S$-th term. Using the Taylor expansion of $\operatorname{arctanh} x$, we have

$$
Q^{(n)}(S, i / 2)=(-i)^{n} \sum_{\substack{i_{1}, \ldots, i_{n} \\ i_{1}+\ldots+i_{n} \leqslant \frac{S-n}{2}}} \prod_{k=1}^{n} \frac{1}{i_{k}+\frac{1}{2}}
$$

After a little algebra we obtain

$$
i^{n} Q^{(n)}(S, i / 2)=\sum_{N=0}^{S-n} \frac{n\left[1+(-1)^{N}\right]}{N+n} i^{n-1} Q^{(n-1)}(N+n-1, i / 2) .
$$

Since $Q^{(n)}(S, i / 2)=0$ for $S<n$, we can replace the lower limit with $-n+1$ and then shift $N \rightarrow N-n$. Then we have

$$
\frac{i^{n}}{n !} Q^{(n)}(S, i / 2)=\sum_{N=1}^{S} \frac{1+(-1)^{N+n}}{N} \frac{i^{n-1}}{(n-1) !} Q^{(n-1)}(N+n-1, i / 2) .
$$

Finally, we obtain

$$
Q^{(n)}(S, i / 2)=(-i)^{n} n ! B_{n}(S)
$$

where $B_{0}(S)=1, B_{1}(S)=H_{1}(S)-H_{-1}(S)$, and $B_{n>1}$ is defined recursively by the symbolic formula

$$
B_{n}=\left(O_{1}+(-1)^{n} O_{-1}\right) B_{n-1},
$$

where $O_{ \pm 1}$ is a linear operator prepending index \pm 1 to harmonic sums, i.e. $O_{ \pm 1} H_{\boldsymbol{a}}(S)=$ $H_{ \pm 1, \boldsymbol{a}}(S)$. In particular, we have

$$
\begin{aligned}
B_{2}= & \left(O_{1}+O_{-1}\right) B_{1}=H_{1,1}+H_{-1,1}-H_{1,-1}-H_{-1,-1}, \\
B_{3}=\left(O_{1}-O_{-1}\right) B_{2}= & H_{1,1,1}+H_{1,-1,1}-H_{1,1,-1}-H_{1,-1,-1} \\
& -H_{-1,1,1}-H_{-1,-1,1}+H_{-1,1,-1}+H_{-1,-1,-1},
\end{aligned}
$$

where we omitted the argument $S$ for brevity. 


\section{Mellin transformations of arising functions}

Let us present inverse Mellin images of the functions which arise in $z$-space.

$$
\begin{aligned}
\mathcal{M}\left[w^{S} \log |w|\right]= & Q(S, u)\left[\gamma_{E}+\log 2-H_{1}(S)-i \eta_{1}\left(u+\frac{i}{2}\right)-\sinh (\pi u) \eta_{-1}\left(u+\frac{i}{2}\right)\right] \\
& -i \sinh (\pi u) \sum_{k=1}^{S} \frac{1-(-1)^{k}}{2 S-k+1} Q(S-k, u)+\sum_{k=1}^{S} \frac{1+(-1)^{k}}{k} Q(S-k, u),
\end{aligned}
$$

where $w=\bar{z}-z$. Note that the combination $f(u)=-i \eta_{1}\left(u+\frac{i}{2}\right)-\sinh (\pi u) \eta_{-1}\left(u+\frac{i}{2}\right)$ appearing in the above formula is an even function, $f(u)=f(-u)$.

Similarly we obtained

$$
\begin{aligned}
\mathcal{M}\left[w^{S} \log (1-w)\right]= & Q(S, u)\left[\gamma_{E}+\log 2-i \eta_{1}\left(-u+\frac{i}{2}\right)-H_{1}(S)\right] \\
& +\sum_{k=1}^{S} \frac{(-1)^{k}}{k} Q(S-k, u)
\end{aligned}
$$

and

$$
\mathcal{M}\left[w^{S} \log (1+w)\right]=Q(S, u)\left[\gamma_{E}+\log 2-i \eta_{1}\left(u+\frac{i}{2}\right)-H_{1}(S)\right]+\sum_{k=1}^{S} \frac{1}{k} Q(S-k, u) .
$$

During the calculation we also needed the expression for inverse Mellin transform of $\mathcal{P}_{1}\left(u+\frac{i}{2}\right) Q(S, u)=\pi \tanh (\pi u) Q(S, u):$

$$
\mathcal{M}^{-1}[\pi \tanh (\pi u) Q(S, u)]=i \ln ((1+w) /(1-w)) w^{S}-i \sum_{k=1}^{S} \frac{1-(-1)^{k}}{k} w^{S-k} .
$$

Open Access. This article is distributed under the terms of the Creative Commons Attribution License (CC-BY 4.0), which permits any use, distribution and reproduction in any medium, provided the original author(s) and source are credited.

\section{References}

[1] G. 't Hooft, A planar diagram theory for strong interactions, Nucl. Phys. B 72 (1974) 461 [INSPIRE].

[2] J.M. Maldacena, The large $N$ limit of superconformal field theories and supergravity, Int. $J$. Theor. Phys. 38 (1999) 1113 [hep-th/9711200] [INSPIRE].

[3] S.S. Gubser, I.R. Klebanov and A.M. Polyakov, Gauge theory correlators from noncritical string theory, Phys. Lett. B 428 (1998) 105 [hep-th/9802109] [INSPIRE].

[4] E. Witten, Anti-de Sitter space and holography, Adv. Theor. Math. Phys. 2 (1998) 253 [hep-th/9802150] [INSPIRE]. 
[5] N. Beisert et al., Review of AdS/CFT integrability: an overview, Lett. Math. Phys. 99 (2012) 3 [arXiv: 1012.3982] [INSPIRE].

[6] D. Bombardelli et al., An integrability primer for the gauge-gravity correspondence: an introduction, J. Phys. A 49 (2016) 320301 [arXiv: 1606.02945] [INSPIRE].

[7] S.J. van Tongeren, Integrability of the $A d S_{5} \times S^{5}$ superstring and its deformations, J. Phys. A 47 (2014) 433001 [arXiv:1310.4854] [INSPIRE].

[8] M. de Leeuw, A.C. Ipsen, C. Kristjansen and M. Wilhelm, Introduction to integrability and one-point functions in $\mathcal{N}=4 S Y M$ and its defect cousin, in Les Houches Summer School: Integrability: From Statistical Systems to Gauge Theory, June 6-July, Les Houches, France (2017), arXiv: 1708.02525 [INSPIRE].

[9] N. Gromov, Introduction to the spectrum of $N=4$ SYM and the quantum spectral curve, arXiv: 1708.03648 [INSPIRE].

[10] S. Komatsu, Lectures on three-point functions in $N=4$ supersymmetric Yang-Mills theory, arXiv: 1710.03853 [INSPIRE].

[11] O. Aharony, O. Bergman, D.L. Jafferis and J. Maldacena, $N=6$ superconformal Chern-Simons-matter theories, M2-branes and their gravity duals, JHEP 10 (2008) 091 [arXiv:0806.1218] [INSPIRE].

[12] M. Staudacher, The factorized S-matrix of CFT/AdS, JHEP 05 (2005) 054 [hep-th/0412188] [INSPIRE].

[13] G. Arutyunov, S. Frolov and M. Staudacher, Bethe ansatz for quantum strings, JHEP 10 (2004) 016 [hep-th/0406256] [INSPIRE].

[14] N. Beisert, The SU(2|2) dynamic S-matrix, Adv. Theor. Math. Phys. 12 (2008) 945 [hep-th/0511082] [INSPIRE].

[15] N. Beisert, The analytic Bethe ansatz for a chain with centrally extended su(2|2) Symmetry, J. Stat. Mech. 01 (2007) P01017 [nlin/0610017].

[16] N. Beisert, B. Eden and M. Staudacher, Transcendentality and crossing, J. Stat. Mech. 0701 (2007) P01021 [hep-th/0610251] [INSPIRE].

[17] R.A. Janik, The AdS $S_{5} \times S^{5}$ superstring worldsheet $S$-matrix and crossing symmetry, Phys. Rev. D 73 (2006) 086006 [hep-th/0603038] [INSPIRE].

[18] G. Arutyunov and S. Frolov, On $A d S_{5} \times S^{5}$ string S-matrix, Phys. Lett. B 639 (2006) 378 [hep-th/0604043] [INSPIRE].

[19] G. Arutyunov, S. Frolov and M. Zamaklar, The Zamolodchikov-Faddeev algebra for $A d S_{5} \times S^{5}$ superstring, JHEP 04 (2007) 002 [hep-th/0612229] [INSPIRE].

[20] C. Ahn and R.I. Nepomechie, $N=6$ super Chern-Simons theory S-matrix and all-loop Bethe ansatz equations, JHEP 09 (2008) 010 [arXiv:0807.1924] [INSPIRE].

[21] J.A. Minahan and K. Zarembo, The Bethe ansatz for $N=4$ super-Yang-Mills, JHEP 03 (2003) 013 [hep-th/0212208] [INSPIRE].

[22] N. Beisert and M. Staudacher, The N=4 SYM integrable super spin chain, Nucl. Phys. B 670 (2003) 439 [hep-th/0307042] [INSPIRE].

[23] N. Beisert and M. Staudacher, Long-range PSU $(2,2 \mid 4)$ Bethe Ansatze for gauge theory and strings, Nucl. Phys. B 727 (2005) 1 [hep-th/0504190] [INSPIRE]. 
[24] J.A. Minahan and K. Zarembo, The Bethe ansatz for superconformal Chern-Simons, JHEP 09 (2008) 040 [arXiv:0806.3951] [INSPIRE].

[25] D. Gaiotto, S. Giombi and X. Yin, Spin chains in $N=6$ superconformal Chern-Simons-matter theory, JHEP 04 (2009) 066 [arXiv: 0806.4589] [INSPIRE].

[26] N. Gromov and P. Vieira, The all loop AdS $4 / C F T_{3}$ Bethe ansatz, JHEP 01 (2009) 016 [arXiv:0807.0777] [INSPIRE].

[27] N. Gromov, V. Kazakov and P. Vieira, Exact spectrum of anomalous dimensions of planar $N=4$ supersymmetric Yang-Mills theory, Phys. Rev. Lett. 103 (2009) 131601 [arXiv: 0901.3753] [INSPIRE].

[28] D. Bombardelli, D. Fioravanti and R. Tateo, Thermodynamic Bethe Ansatz for planar AdS/CFT: a proposal, J. Phys. A 42 (2009) 375401 [arXiv:0902.3930] [InSPIRE].

[29] N. Gromov, V. Kazakov, A. Kozak and P. Vieira, Exact spectrum of anomalous dimensions of planar $N=4$ supersymmetric Yang-Mills theory: TBA and excited states, Lett. Math. Phys. 91 (2010) 265 [arXiv:0902.4458] [INSPIRE].

[30] G. Arutyunov and S. Frolov, Thermodynamic Bethe ansatz for the $A d S_{5} \times S^{5}$ mirror model, JHEP 05 (2009) 068 [arXiv:0903.0141] [INSPIRE].

[31] A. Cavaglia, D. Fioravanti and R. Tateo, Extended Y-system for the $A d S_{5} / C F T_{4}$ correspondence, Nucl. Phys. B 843 (2011) 302 [arXiv:1005.3016] [INSPIRE].

[32] J. Balog and A. Hegedus, $A d S_{5} \times S^{5}$ mirror TBA equations from $Y$-system and discontinuity relations, JHEP 08 (2011) 095 [arXiv: 1104.4054] [INSPIRE].

[33] N. Gromov, V. Kazakov, S. Leurent and Z. Tsuboi, Wronskian solution for AdS/CFT $Y$-system, JHEP 01 (2011) 155 [arXiv:1010.2720] [INSPIRE].

[34] N. Gromov, V. Kazakov, S. Leurent and D. Volin, Solving the AdS/CFT Y-system, JHEP 07 (2012) 023 [arXiv: 1110.0562] [INSPIRE].

[35] D. Bombardelli, D. Fioravanti and R. Tateo, TBA and $Y$-system for planar $A d S_{4} / C F T_{3}$, Nucl. Phys. B 834 (2010) 543 [arXiv:0912.4715] [INSPIRE].

[36] N. Gromov and F. Levkovich-Maslyuk, Y-system, TBA and quasi-classical strings in $A d S_{4} \times C P^{3}, J H E P 06$ (2010) 088 [arXiv:0912.4911] [INSPIRE].

[37] A. Cavaglia, D. Fioravanti and R. Tateo, Discontinuity relations for the $A d S_{4} / C F T_{3}$ correspondence, Nucl. Phys. B 877 (2013) 852 [arXiv: 1307.7587] [InSPIRE].

[38] D. Correa, J. Maldacena and A. Sever, The quark anti-quark potential and the cusp anomalous dimension from a TBA equation, JHEP 08 (2012) 134 [arXiv:1203.1913] [INSPIRE].

[39] N. Drukker, Integrable Wilson loops, JHEP 10 (2013) 135 [arXiv:1203.1617] [inSPIRE].

[40] N. Gromov and F. Levkovich-Maslyuk, Quantum spectral curve for a cusped Wilson line in $\mathcal{N}=4$ SYM, JHEP 04 (2016) 134 [arXiv: 1510.02098] [INSPIRE].

[41] N. Gromov and F. Levkovich-Maslyuk, Quark-anti-quark potential in $\mathcal{N}=4 S Y M$, JHEP 12 (2016) 122 [arXiv:1601.05679] [INSPIRE].

[42] L.F. Alday, D. Gaiotto and J. Maldacena, Thermodynamic bubble ansatz, JHEP 09 (2011) 032 [arXiv:0911.4708] [INSPIRE]. 
[43] L.F. Alday, J. Maldacena, A. Sever and P. Vieira, Y-system for scattering amplitudes, J. Phys. A 43 (2010) 485401 [arXiv: 1002.2459] [InSPIRE].

[44] L.F. Alday et al., An operator product expansion for polygonal null Wilson loops, JHEP 04 (2011) 088 [arXiv: 1006.2788] [INSPIRE].

[45] B. Basso, A. Sever and P. Vieira, Spacetime and flux tube S-matrices at finite coupling for $N=4$ supersymmetric Yang-Mills theory, Phys. Rev. Lett. 111 (2013) 091602 [arXiv:1303.1396] [INSPIRE].

[46] B. Basso, J. Caetano, L. Cordova, A. Sever and P. Vieira, OPE for all helicity amplitudes, JHEP 08 (2015) 018 [arXiv: 1412.1132] [INSPIRE].

[47] D. Fioravanti, S. Piscaglia and M. Rossi, Asymptotic Bethe Ansatz on the GKP vacuum as a defect spin chain: scattering, particles and minimal area Wilson loops, Nucl. Phys. B 898 (2015) 301 [arXiv: 1503.08795] [INSPIRE].

[48] B. Basso, S. Caron-Huot and A. Sever, Adjoint BFKL at finite coupling: a short-cut from the collinear limit, JHEP 01 (2015) 027 [arXiv:1407.3766] [INSPIRE].

[49] M. Alfimov, N. Gromov and V. Kazakov, QCD Pomeron from AdS/CFT quantum spectral curve, JHEP 07 (2015) 164 [arXiv: 1408.2530] [INSPIRE].

[50] N. Gromov, F. Levkovich-Maslyuk and G. Sizov, Pomeron eigenvalue at three loops in $\mathcal{N}=4$ supersymmetric Yang-Mills theory, Phys. Rev. Lett. 115 (2015) 251601 [arXiv: 1507.04010] [INSPIRE].

[51] B. Basso, S. Komatsu and P. Vieira, Structure constants and integrable bootstrap in planar $N=4 S Y M$ theory, arXiv:1505.06745 [INSPIRE].

[52] B. Basso, V. Goncalves and S. Komatsu, Structure constants at wrapping order, JHEP 05 (2017) 124 [arXiv: 1702 . 02154] [inSPIRE].

[53] Y. Jiang, S. Komatsu, I. Kostov and D. Serban, Clustering and the three-point function, J. Phys. A 49 (2016) 454003 [arXiv: 1604.03575] [InSPIRE].

[54] I. Balitsky, V. Kazakov and E. Sobko, Structure constant of twist-2 light-ray operators in the Regge limit, Phys. Rev. D 93 (2016) 061701 [arXiv: 1506.02038] [INSPIRE].

[55] M. de Leeuw, C. Kristjansen and K. Zarembo, One-point functions in defect CFT and integrability, JHEP 08 (2015) 098 [arXiv:1506.06958] [INSPIRE].

[56] I. Buhl-Mortensen, M. de Leeuw, C. Kristjansen and K. Zarembo, One-point functions in AdS/dCFT from matrix product states, JHEP 02 (2016) 052 [arXiv:1512.02532] [INSPIRE].

[57] I. Buhl-Mortensen et al., One-loop one-point functions in gauge-gravity dualities with defects, Phys. Rev. Lett. 117 (2016) 231603 [arXiv:1606.01886] [INSPIRE].

[58] N. Gromov, V. Kazakov, S. Leurent and D. Volin, Quantum spectral curve for planar $\mathcal{N}=4$ Super-Yang-Mills theory, Phys. Rev. Lett. 112 (2014) 011602 [arXiv:1305.1939] [INSPIRE].

[59] N. Gromov, V. Kazakov, S. Leurent and D. Volin, Quantum spectral curve for arbitrary state/operator in $A d S_{5} / C F T_{4}, J H E P O 9$ (2015) 187 [arXiv:1405.4857] [INSPIRE].

[60] V. Kazakov, S. Leurent and D. Volin, T-system on T-hook: grassmannian solution and twisted quantum spectral curve, JHEP 12 (2016) 044 [arXiv:1510.02100] [INSPIRE].

[61] C. Marboe and D. Volin, The full spectrum of $A d S_{5} / C F T_{4}$ I: representation theory and one-loop Q-system, J. Phys. A 51 (2018) 165401 [arXiv:1701.03704] [INSPIRE]. 
[62] A. Cavagli, D. Fioravanti, N. Gromov and R. Tateo, Quantum spectral curve of the $\mathcal{N}=6$ supersymmetric Chern-Simons theory, Phys. Rev. Lett. 113 (2014) 021601 [arXiv:1403.1859] [INSPIRE].

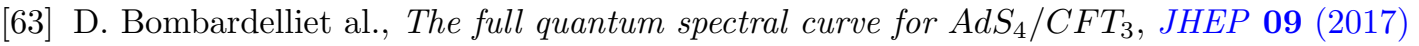
140 [arXiv: 1701.00473] [INSPIRE].

[64] C. Marboe and D. Volin, Quantum spectral curve as a tool for a perturbative quantum field theory, Nucl. Phys. B 899 (2015) 810 [arXiv:1411.4758] [INSPIRE].

[65] L. Anselmetti et al., 12 loops and triple wrapping in ABJM theory from integrability, JHEP 10 (2015) 117 [arXiv: 1506.09089] [INSPIRE].

[66] L.N. Lipatov, Next-to-leading corrections to the BFKL equation and the effective action for high energy processes in QCD, Nucl. Phys. Proc. Suppl. 99A (2001) 175 [inSPIRE].

[67] A.V. Kotikov and L.N. Lipatov, NLO corrections to the BFKL equation in $Q C D$ and in supersymmetric gauge theories, Nucl. Phys. B 582 (2000) 19 [hep-ph/0004008] [INSPIRE].

[68] A.V. Kotikov, L.N. Lipatov and V.N. Velizhanin, Anomalous dimensions of Wilson operators in $N=4$ SYM theory, Phys. Lett. B 557 (2003) 114 [hep-ph/0301021] [INSPIRE].

[69] A.V. Kotikov and L.N. Lipatov, DGLAP and BFKL evolution equations in the $N=4$ supersymmetric gauge theory, talk given at the $35^{\text {th }}$ Annual Winter School on Nuclear and Particle Physics, February 19-25, Repino, Russia (2001), hep-ph/0112346 [INSPIRE].

[70] A.V. Kotikov and L.N. Lipatov, DGLAP and BFKL equations in the $N=4$ supersymmetric gauge theory, Nucl. Phys. B 661 (2003) 19 [Erratum ibid. B 685 (2004) 405] [hep-ph/0208220] [INSPIRE].

[71] A.V. Kotikov, L.N. Lipatov, A.I. Onishchenko and V.N. Velizhanin, Three loop universal anomalous dimension of the Wilson operators in $N=4$ SUSY Yang-Mills model, Phys. Lett. B 595 (2004) 521 [Erratum ibid. B 632 (2006) 754] [hep-th/0404092] [INSPIRE].

[72] A.V. Kotikov et al., Dressing and wrapping, J. Stat. Mech. 0710 (2007) P10003 [arXiv:0704.3586] [INSPIRE].

[73] T. Lukowski, A. Rej and V.N. Velizhanin, Five-loop anomalous dimension of twist-two operators, Nucl. Phys. B 831 (2010) 105 [arXiv: 0912.1624] [INSPIRE].

[74] V.N. Velizhanin, Six-loop anomalous dimension of twist-three operators in $N=4 S Y M$, JHEP 11 (2010) 129 [arXiv:1003.4717] [INSPIRE].

[75] C. Marboe, V. Velizhanin and D. Volin, Six-loop anomalous dimension of twist-two operators in planar $\mathcal{N}=4$ SYM theory, JHEP 07 (2015) 084 [arXiv:1412.4762] [INSPIRE].

[76] C. Marboe and V. Velizhanin, Twist-2 at seven loops in planar $\mathcal{N}=4$ SYM theory: full result and analytic properties, JHEP 11 (2016) 013 [arXiv:1607.06047] [INSPIRE].

[77] M. Beccaria and G. Macorini, QCD properties of twist operators in the $N=6$ Chern-Simons theory, JHEP 06 (2009) 008 [arXiv:0904.2463] [INSPIRE].

[78] J.A. Minahan, O. Ohlsson Sax and C. Sieg, Magnon dispersion to four loops in the ABJM and ABJ models, J. Phys. A 43 (2010) 275402 [arXiv:0908.2463] [inSPIRE].

[79] J.A. Minahan, O. Ohlsson Sax and C. Sieg, Anomalous dimensions at four loops in $N=6$ superconformal Chern-Simons theories, Nucl. Phys. B 846 (2011) 542 [arXiv:0912.3460] [INSPIRE]. 
[80] G. Papathanasiou and M. Spradlin, Two-loop spectroscopy of short ABJM operators, JHEP 02 (2010) 072 [arXiv:0911.2220] [INSPIRE].

[81] M. Leoni et al., Superspace calculation of the four-loop spectrum in $N=6$ supersymmetric Chern-Simons theories, JHEP 12 (2010) 074 [arXiv:1010.1756] [INSPIRE].

[82] M. Beccaria, F. Levkovich-Maslyuk and G. Macorini, On wrapping corrections to GKP-like operators, JHEP 03 (2011) 001 [arXiv:1012.2054] [INSPIRE].

[83] M.A. Bandres, A.E. Lipstein and J.H. Schwarz, Studies of the ABJM theory in a formulation with manifest SU(4) R-symmetry, JHEP 09 (2008) 027 [arXiv:0807.0880] [INSPIRE].

[84] T. Klose, Review of AdS/CFT integrability, Chapter IV.3: $N=6$ Chern-Simons and strings on $A d S_{4} \times C P^{3}$, Lett. Math. Phys. 99 (2012) 401 [arXiv:1012.3999] [INSPIRE].

[85] G. Grignani, T. Harmark and M. Orselli, The $\mathrm{SU}(2) \times \mathrm{SU}(2)$ sector in the string dual of $N=6$ superconformal Chern-Simons theory, Nucl. Phys. B 810 (2009) 115 [arXiv: 0806.4959] [INSPIRE].

[86] N. Gromov and G. Sizov, Exact slope and interpolating functions in $n=6$ supersymmetric Chern-Simons theory, Phys. Rev. Lett. 113 (2014) 121601 [arXiv:1403.1894] [INSPIRE].

[87] A. Cavaglià, N. Gromov and F. Levkovich-Maslyuk, On the exact interpolating function in ABJ theory, JHEP 12 (2016) 086 [arXiv:1605.04888] [INSPIRE].

[88] J. Ablinger, A computer algebra toolbox for harmonic sums related to particle physics, Ph.D. thesis, Linz University, Linz, Austria (2009), arXiv:1011.1176 [INSPIRE].

[89] J. Ablinger, Computer algebra algorithms for special functions in particle physics, Ph.D. thesis, Linz University, Linz, Austria (2012), arXiv: 1305.0687 [INSPIRE].

[90] J. Ablinger, J. Blümlein and C. Schneider, Analytic and algorithmic aspects of generalized harmonic sums and polylogarithms, J. Math. Phys. 54 (2013) 082301 [arXiv:1302.0378] [INSPIRE].

[91] J. Ablinger, J. Blümlein and C. Schneider, Harmonic sums and polylogarithms generated by cyclotomic polynomials, J. Math. Phys. 52 (2011) 102301 [arXiv:1105.6063] [INSPIRE].

[92] J. Blumlein, Structural relations of harmonic sums and Mellin transforms up to weight $w=5$, Comput. Phys. Commun. 180 (2009) 2218 [arXiv:0901.3106] [INSPIRE].

[93] E. Remiddi and J.A.M. Vermaseren, Harmonic polylogarithms, Int. J. Mod. Phys. A 15 (2000) 725 [hep-ph/9905237] [INSPIRE].

[94] J.A.M. Vermaseren, Harmonic sums, Mellin transforms and integrals, Int. J. Mod. Phys. A 14 (1999) 2037 [hep-ph/9806280] [INSPIRE].

[95] L.N. Lipatov, Asymptotic behavior of multicolor QCD at high energies in connection with exactly solvable spin models, JETP Lett. 59 (1994) 596 [hep-th/9311037] [INSPIRE].

[96] L.D. Faddeev and G.P. Korchemsky, High-energy QCD as a completely integrable model, Phys. Lett. B 342 (1995) 311 [hep-th/9404173] [INSPIRE].

[97] A.V. Kotikov, A. Rej and S. Zieme, Analytic three-loop solutions for $N=4 S Y M$ twist operators, Nucl. Phys. B 813 (2009) 460 [arXiv:0810.0691] [INSPIRE].

[98] M. Beccaria, A.V. Belitsky, A.V. Kotikov and S. Zieme, Analytic solution of the multiloop Baxter equation, Nucl. Phys. B 827 (2010) 565 [arXiv:0908.0520] [INSPIRE]. 
[99] J. Fleischer, A.V. Kotikov and O.L. Veretin, Analytic two loop results for selfenergy type and vertex type diagrams with one nonzero mass, Nucl. Phys. B 547 (1999) 343 [hep-ph/9808242] [INSPIRE].

[100] A.V. Kotikov, The property of maximal transcendentality in the $N=4$ supersymmetric Yang-Mills, in Subtleties in quantum field theory: Lev Lipatov Festschrift, D. Diakonov ed., St. Petersburg Nucl. Phys. Inst., Russia (2001), arXiv: 1005.5029 [INSPIRE].

[101] A.V. Kotikov, The property of maximal transcendentality: calculation of Feynman integrals, Theor. Math. Phys. 190 (2017) 391 [arXiv:1601.00486] [INSPIRE].

[102] S. Moch, P. Uwer and S. Weinzierl, Nested sums, expansion of transcendental functions and multiscale multiloop integrals, J. Math. Phys. 43 (2002) 3363 [hep-ph/0110083] [INSPIRE].

[103] Yu.L. Dokshitzer, G. Marchesini and G.P. Salam, Revisiting parton evolution and the large-x limit, Phys. Lett. B 634 (2006) 504 [hep-ph/0511302] [INSPIRE].

[104] Yu. L. Dokshitzer and G. Marchesini, $N=4$ SUSY Yang-Mills: three loops made simple(r), Phys. Lett. B 646 (2007) 189 [hep-th/0612248] [INSPIRE].

[105] A.B. Zamolodchikov, 'Fishnet' diagrams as a completely integrable system, Phys. Lett. B 97 (1980) 63.

[106] D. Chicherin, S. Derkachov and A.P. Isaev, Conformal group: R-matrix and star-triangle relation, JHEP 04 (2013) 020 [arXiv: 1206.4150] [INSPIRE].

[107] O. Gurdogan and V. Kazakov, New integrable 4D quantum field theories from strongly deformed planar $\mathcal{N}=4$ supersymmetric Yang-Mills theory, Phys. Rev. Lett. 117 (2016) 201602 [arXiv: 1512.06704] [INSPIRE].

[108] J. Caetano, O. Gurdogan and V. Kazakov, Chiral limit of $\mathcal{N}=4 S Y M$ and ABJM and integrable Feynman graphs, JHEP 03 (2018) 077 [arXiv: 1612.05895] [INSPIRE].

[109] D. Chicherin et al., Yangian symmetry for bi-scalar loop amplitudes, JHEP 05 (2018) 003 [arXiv: 1704.01967] [INSPIRE].

[110] B. Basso and L.J. Dixon, Gluing ladder Feynman diagrams into fishnets, Phys. Rev. Lett. 119 (2017) 071601 [arXiv:1705.03545] [INSPIRE].

[111] N. Gromov et al., Integrability of conformal fishnet theory, JHEP 01 (2018) 095 [arXiv: 1706. 04167] [INSPIRE].

[112] D. Chicherin et al., Yangian symmetry for fishnet Feynman graphs, Phys. Rev. D 96 (2017) 121901 [arXiv: 1708.00007] [INSPIRE]. 